\title{
Enantioselective Allylation of Ketone-Derived Benzoylhydrazones: Practical Synthesis of Tertiary Carbinamines
}

\author{
Richard Berger, Keiko Duff, and James L. Leighton* \\ Department of Chemistry, Columbia University, New York, New York, 10027 \\ Supporting Information
}

General Information. All reactions were carried out under an atmosphere of nitrogen in flame- or oven-dried glassware with magnetic stirring unless otherwise indicated. Degassed solvents were purified by passage through an activated alumina column. Toluene, hexanes, methanol and glacial acetic acid were purchased from Fisher and used as received. Anhydrous chloroform (stabilized with amylenes), anhydrous methylene chloride, benzoic hydrazide and all ketone reagents were purchased from Aldrich and used as received. ${ }^{1} \mathrm{H}$ NMR spectra were recorded on a Bruker DPX-400 (400 MHz) spectrometer and are reported in ppm from either $\mathrm{CDCl}_{3}$ internal standard $(7.26 \mathrm{ppm})$ or DMSO- $d_{6}$ internal standard $(2.49$ ppm). Data are reported as follows: $(\mathrm{s}=$ singlet, br $\mathrm{s}=$ broad singlet, $\mathrm{d}=$ doublet, $\mathrm{t}=$ triplet, $\mathrm{q}=$ quartet, quin $=$ quintet, sep $=$ septet, $\mathrm{m}=$ multiplet, $\mathrm{dd}=$ doublet of doublets, $\mathrm{td}=$ triplet of doublets, $\mathrm{tt}=$ triplet of triplets, $\mathrm{dq}=$ doublet of quartets, $\mathrm{ddt}=$ doublet of doublet of triplets; coupling constant(s) in $\mathrm{Hz}$; integration; assignment). Proton decoupled ${ }^{13} \mathrm{C}$ NMR spectra were recorded on a Bruker DPX-400 (100 $\mathrm{MHz}$ ) and are reported in ppm from either $\mathrm{CDCl}_{3}$ internal standard (77.0 ppm), DMSO- $d_{6}$ internal standard (39.5 ppm) or $\mathrm{CD}_{3} \mathrm{OD}$ internal standard (49.0 ppm). Infrared spectra were recorded on a Perkin Elmer Paragon 1000 FT-IR spectrometer. Optical rotations were recorded on a Jasco DIP-1000 digital polarimeter.

Preparation of reagent $(S, S)-1$ : The procedure for preparation and characterization data for this reagent may be found in the supporting information of our earlier work. ${ }^{1}$

(1) (a) Kinnard, J. W. A.; Ng, P. Y.; Kubota, K.; Wang, X.; Leighton, J. L. J. Am. Chem. Soc. 2002, 124, 7920-7921. (b) Berger, R.; Rabbat, P. M. A.; Leighton, J. L. J. Am. Chem. Soc. 2003, 125, 79207921. 
Preparation of benzoic acid (1-phenyl-ethylidene)-hydrazide (Table 1, Entry 1): Benzoic hydrazide $(11.3 \mathrm{~g}, 83.3 \mathrm{mmol})$ was added to a stirred solution of acetophenone $(10.0 \mathrm{~g}, 83.3 \mathrm{mmol})$ and glacial acetic acid $(0.500 \mathrm{~mL}, 8.73 \mathrm{mmol})$ in methanol:hexanes $(1: 5)(70 \mathrm{~mL})$. The resulting suspension was heated to reflux for $12 \mathrm{hr}$ during which time all solids dissolved. After cooling to ambient temperature the mixture was concentrated and the residue recrystallized from toluene to yield benzoic acid (1-phenyl-ethylidene)-hydrazide (16.0 g, 80\%) as a white solid.

Note: This procedure was used for the preparation of all benzoylhydrazones. Characterization data for substrates not previously reported are listed below.

Benzoic acid (1,2-diphenyl-ethylidene)-hydrazide (Table 1, Entry 3): Recrystallized from toluene (88\% yield): ${ }^{1} \mathrm{H}$ NMR (400 MHz, DMSO-d $d_{6}$ ) 10.99 (br s, 1H, N-H), 7.84 (br s, 2H, Ar-H), 7.72-7.74 (m, 2H, Ar-H), 7.45-7.56 (m, 3H, Ar-H), 7.37 (br s, 3H, Ar-H), 7.19-7.30 (m, 2H, Ar-H), 7.15-7.18 (m, 3H, Ar-H), 4.40 (s, 2H, CH$-\mathrm{Ph}),{ }^{13} \mathrm{C}$ NMR (100 MHz, $\left.\mathrm{CDCl}_{3}\right) \square 163.1,135.1,137.4,133.9,133.0,131.6$, 129.4, 128.3, 127.8, 127.4, 126.7, 34.2; IR (KBr): 3345, 3081, 3058, 3026, 2941, 2873, 1658, 1600, 1580, $1520,1484,1457,1265,1131,1101,1075 \mathrm{~cm}^{-1}$; LRMS (FAB+) calcd for $\mathrm{C}_{21} \mathrm{H}_{18} \mathrm{~N}_{2} \mathrm{O} 314$, found $(\mathrm{M}+\mathrm{H})^{+}$ 315.

Benzoic acid (2-methyl-1-phenyl-propylidene)-hydrazide (Table 1, Entry 4): Recrystallized from toluene (68\% yield): ${ }^{1} \mathrm{H}$ NMR (400 MHz, DMSO- $d_{6}$ ) 10:1 mixture of rotamers: (major) $\square 9.63$ (br s, $1 \mathrm{H}$, N-H), 7.33-7.81 (m, 10H, Ar-H), 2.90 (br s, 1H, $\left.\left(\mathrm{CH}_{3}\right)_{2} \mathrm{C}-\mathbf{H}\right), 1.07-1.14$ (m, 6H, CH-(CH$\left.)_{2}\right),($ minor) $\square$ 10.82 (br s, 1H, N-H), 7.33-7.81 (m, 10H, Ar-H), $3.61\left(\mathrm{~m}, 1 \mathrm{H},\left(\mathrm{CH}_{3}\right)_{2} \mathrm{C}-\mathbf{H}\right), 1.07-1.14(\mathrm{~m}, 6 \mathrm{H}, \mathrm{CH}-$ $\left.\left(\mathrm{CH}_{3}\right)_{2}\right) ;{ }^{13} \mathrm{C} \mathrm{NMR}\left(100 \mathrm{MHz}, \mathrm{CDCl}_{3}\right) \square 163.4,163.1,133.3,132.3,131.7,130.3,129.5,128.6,127.4$, 127.1, 126.8, 36.5, 19.9; IR (KBr): 3359, 2969, 1670, 1505, 1480, 1464, 1440, 1307, 1292, 1259, 1118 , $1073 \mathrm{~cm}^{-1}$; LRMS (FAB+) calcd for $\mathrm{C}_{17} \mathrm{H}_{18} \mathrm{~N}_{2} \mathrm{O} 266$, found $(\mathrm{M}+\mathrm{H})^{+} 267$.

(Benzoyl-hydrazono)-phenyl-acetic acid methyl ester (Table 1, Entry 5): Recrystallized from toluene (70\% yield): ${ }^{1} \mathrm{H}$ NMR (400 MHz, $\mathrm{CDCl}_{3}$ ) $\square 13.3$ (br s, 1H, N-H), 7.91-8.01 (m, 2H, Ar-H), 7.627-68 (m, 2H, Ar-H), 7.50-7.62 (m, 3H, Ar-H), 7.35-7.43 (m, 3H, Ar-H), 3.95 (s, 3H, $\left.\mathrm{CO}_{2} \mathrm{CH}_{3}\right)$; ${ }^{13} \mathrm{C}$ NMR $\left(100 \mathrm{MHz}, \mathrm{CDCl}_{3}\right) \square 162.9,138.9,134.0,132.3,132.1,129.1,128.7,128.5,127.8,127.3,52.8$; IR (KBr): 
3264, 3060, 3026, 2953, 1701, 1688, 1600, 1578, 1535, 1506, 1475, 1242, $1145 \mathrm{~cm}^{-1}$; LRMS (FAB+) calcd for $\mathrm{C}_{16} \mathrm{H}_{14} \mathrm{~N}_{2} \mathrm{O}_{3} 282$, found $(\mathrm{M}+\mathrm{H})^{+} 283$.

Benzoic acid (1-furan-2-yl-ethylidene)-hydrazide (Table 1, Entry 10): Recrystallized from toluene (77\% yield): ${ }^{1} \mathrm{H}$ NMR (400 MHz, DMSO- $d_{6}$ ) $\square 10.67$ (br s, 1H, N-H), 7.84-7.86 (m, 2H, Ar-H), 7.79 (br s, 1H, Ar-H), 7.47-7.58 (m, 3H, Ar-H), 6.96 (br s, 1H, Ar-H), 6.60 (br s, 1H, Ar-H), 2.27 (s, 3H, N=C$\left.\mathrm{CH}_{3}\right) ;{ }^{13} \mathrm{C}$ NMR $\left(100 \mathrm{MHz}, \mathrm{DMSO}-d_{6}\right) \square 151.4,144.3,133.7,131.1,127.9,127.5,111.7,111.3,13.9 ;$ IR (KBr): 3244, 3115, 3035, 1652, 1603, 1573, 1525, 1482, 1318, 1279, 1164, 1141, 1110, 1075, $1026 \mathrm{~cm}^{-1}$; LRMS (FAB+) calcd for $\mathrm{C}_{13} \mathrm{H}_{12} \mathrm{~N}_{2} \mathrm{O}_{2} 228$, found $(\mathrm{M}+\mathrm{H})^{+} 229$.

Benzoic acid (1-thiophen-2-yl-ethylidene)-hydrazide (Table 1, Entry 11): Recrystallized from (1:1) EtOAc:toluene (85\% yield): ${ }^{1} \mathrm{H}$ NMR (400 MHz, DMSO- $\left.d_{6}\right) \square 10.75$ (br s, $\left.1 \mathrm{H}, \mathrm{N}-\mathbf{H}\right), 7.85-7.86$ (m, 2H, Ar-H), 7.47-7.59 (m, 5H, Ar-H), 7.10 (br s, 1H, Ar-H), 2.37 (s, 3H, N=C-CH $)$; ${ }^{13} \mathrm{C} \mathrm{NMR} \mathrm{(100} \mathrm{MHz,}$ DMSO- $\left.d_{6}\right) \square 163.6,152.7,143.2,134.0,131.5,129.1,128.4,127.8,127.6,15.0$; IR (KBr): 3209, 3066, $3025,1650,1540,1517,1488,1435,1307,1275,1234,1139,1050 \mathrm{~cm}^{-1}$; LRMS (FAB+) calcd for $\mathrm{C}_{13} \mathrm{H}_{12} \mathrm{~N}_{2} \mathrm{OS} 244$, found $(\mathrm{M}+\mathrm{H})^{+} 245$.

3-[1-(Benzoyl-hydrazono)-ethyl]-indole-1-carboxylic acid tert-butyl ester (Table 1, Entry 12): Recrystallized from toluene (87\% yield): ${ }^{1} \mathrm{H}$ NMR (400 MHz, DMSO- $d_{6}$ ) $\square 10.77$ (br s, $1 \mathrm{H}, \mathrm{N}-\mathbf{H}$ ), 8.76 (br s, 1H, Ar-H), 8.00-8.20 (m, 2H, Ar-H), 7.90-7.92 (m, 2H, Ar-H), 7.50-7.61 (m, 3H, Ar-H), 7.33-7.39 (m, 2H, Ar-H), 2.42 (s, 3H, N=C-CH$), 1.65$ (s, 9H, COC $\left.\left(\mathrm{CH}_{3}\right)_{3}\right) ;{ }^{13} \mathrm{C} \mathrm{NMR}\left(100 \mathrm{MHz}, \mathrm{CDCl}_{3}\right) \square 149.1$, $135.7,133.5,131.7,128.6,127.6,127.0,126.6,125.0,119.8,114.5,84.4$, 28.3, 13.5; IR (KBr): 3271, $3071,2977,2933,1732,1640,1450,1370,1309,1275,1251,1154,1068 \mathrm{~cm}^{-1}$; LRMS (FAB+) calcd for $\mathrm{C}_{22} \mathrm{H}_{23} \mathrm{~N}_{3} \mathrm{O}_{3} 377$, found $(\mathrm{M}+\mathrm{H})^{+} 378$.

Benzoic acid (1-cyclohexyl-ethylidene)-hydrazide (Table 1, Entry 14): Recrystallized from toluene (82\% yield): ${ }^{1} \mathrm{H}$ NMR (400 MHz, DMSO- $d_{6}$ ) $\square 10.32$ (br s, $1 \mathrm{H}, \mathrm{N}-\mathbf{H}$ ), 7.79-7.81 (m, 2H, Ar-H), 7.46-7.52 (m, 3H, Ar-H), 2.23 (br s, 1H, Cy-H), 1.89 (s, 3H, N=C-CH $), 1.63-1.75$ (m, 5H, Cy-H), 1.17-1.31 (m, 5H, Cy-H); ${ }^{13} \mathrm{C}$ NMR (100 MHz, DMSO- $\left.d_{6}\right) \square 163.5,162.7,133.9,130.8,127.9,127.3,46.5,29.7,28.5$, 25.7, 25.6, 14.9; IR (KBr): 3219, 3031, 2928, 2850, 1653, 1628, 1581, 1542, 1446, 1290, 1146, $1129 \mathrm{~cm}^{-}$ ${ }^{1}$; LRMS (FAB+) calcd for $\mathrm{C}_{15} \mathrm{H}_{20} \mathrm{~N}_{2} \mathrm{O} 244$, found $(\mathrm{M}+\mathrm{H})^{+} 245$. 
Benzoic acid $N$-methyl- $N^{\prime}$-(1-phenyl-ethylidene)-hydrazide (Scheme 3, Compound 6): Compound 6 was prepared by the same method as above using benzoic acid $N$-methyl-hydrazide in place of benzoic hydrazide. Recrystallized from toluene (80\% yield): ${ }^{1} \mathrm{H}$ NMR (400 MHz, DMSO- $\left.d_{6}\right) \square 7.70$ (br s, 2H, Ar-H), 7.41-7.47 (br s, 8H, Ar-H), 3.25 (s, 3H, CH$-\mathrm{NCOPh}$ ), 2.30 (br s, 3H, N=C-CH ${ }_{3}$ ); ${ }^{13} \mathrm{C}$ NMR $\left(100{\left.\mathrm{MHz}, \mathrm{CDCl}_{3}\right)}\right) 169.8,136.7,135.2,130.4,129.9,128.1,126.7,36.3$ (br), 17.1; IR (KBr): 3055 , 2948, 1637, 1609, 1573, 1446, 1422, 1346, 1303, 1057, 1023, 794, $716 \mathrm{~cm}^{-1}$; LRMS (FAB+) calcd for $\mathrm{C}_{16} \mathrm{H}_{16} \mathrm{~N}_{2} \mathrm{O} 252$, found $(\mathrm{M}+\mathrm{H})^{+} 253$.

Representative procedure for the enantioselective allylation of benzoylhydrazones with $(S, S)-1$ : To a solution of the benzoylhydrazone $(0.800 \mathrm{mmol})$ in $\mathrm{CHCl}_{3}(5 \mathrm{~mL})$ is added $(S, S)-\mathbf{1}(320 \mathrm{mg}, 1.20$ mmol) and the resulting mixture is stirred at the indicated temperature for $24 \mathrm{~h}$ (see Table 1). The reaction is quenched with methanol $(2 \mathrm{~mL})$, the resulting mixture is stirred for $15 \mathrm{~min}$, and then concentrated. The residue is diluted with EtOAc $(5 \mathrm{~mL})$ and $\mathrm{H}_{2} \mathrm{O}(5 \mathrm{~mL})$, the phases are separated, and the aqueous layer is extracted with EtOAc $(2 \times 5 \mathrm{~mL})$. The combined organic layers are washed with brine $(1 \times 5 \mathrm{~mL})$, dried $\left(\mathrm{MgSO}_{4}\right)$, filtered, and concentrated. Purification by flash chromatography on silica gel affords the pure hydrazide products in the yields and enantioselectivities reported in Table 1.

(R)-Benzoic acid $N^{\prime}$-(1-methyl-1-phenyl-but-3-enyl)-hydrazide (Table 1, Entry 1): $[\square]_{\mathrm{D}}=+78.5^{\circ}$ $\left(c\right.$ 1.0, $\left.\mathrm{CHCl}_{3}\right) ;{ }^{1} \mathrm{H}$ NMR (400 MHz, $\left.\mathrm{CDCl}_{3}\right) \square 7.54-7.60$ (m, 4H, Ar-H), 7.47 (t, $\left.J=7.3 \mathrm{~Hz}, 1 \mathrm{H}, \mathrm{Ar}-\mathbf{H}\right)$, 7.36-7.41 (m, 4H, Ar-H), 7.29 (t, $J=7.3 \mathrm{~Hz}, 1 \mathrm{H}$, Ar-H), 7.08 (br s, 1H, PhCON-H), 5.63-5.72 (m, 1H, $\left.\mathrm{CH}_{2} \mathrm{CH}=\mathrm{CH}_{2}\right), 5.44$ (br s, $\left.1 \mathrm{H}, \mathrm{ArC}-\mathrm{NH}\right), 5.07-5.12\left(\mathrm{~m}, 2 \mathrm{H}, \mathrm{CH}_{2} \mathrm{CH}=\mathrm{CH}_{2}\right), 2.62(\mathrm{dd}, J=13.7,6.6 \mathrm{~Hz}, 1 \mathrm{H}$, one of $\left.\mathrm{CH}_{2} \mathrm{CH}=\mathrm{CH}_{2}\right), 2.52\left(\mathrm{dd}, J=13.7,8.0 \mathrm{~Hz}, 1 \mathrm{H}\right.$, one of $\left.\mathrm{CH}_{2} \mathrm{CH}=\mathrm{CH}_{2}\right), 1.56\left(\mathrm{~s}, 3 \mathrm{H}, \mathrm{ArC}-\mathrm{CH}_{3}\right) ;{ }^{13} \mathrm{C}$ NMR (100 MHz, $\left.\mathrm{CDCl}_{3}\right) \square 166.5,144.2,133.5,132.7,131.5,128.5,128.4,127.0,126.7,126.4,118.4$, 62.4, 46.0, 22.6; IR (thin film) 3275, 3061, 2976, 2929, 1637, 1578, 1542, 1494, 1446, 1374, $1313 \mathrm{~cm}^{-1}$; LRMS (FAB+) calcd for $\mathrm{C}_{18} \mathrm{H}_{20} \mathrm{~N}_{2} \mathrm{O} 280$, found $(\mathrm{M}+\mathrm{H})^{+} 281$.

(R)-Benzoic acid $N^{\prime}$-(1-ethyl-1-phenyl-but-3-enyl)-hydrazide (Table 1, Entry 2): $[\square]_{\mathrm{D}}=-16.7^{\circ}(c$ 2.0, $\mathrm{CHCl}_{3}$ ); ${ }^{1} \mathrm{H} \mathrm{NMR}\left(400 \mathrm{MHz}, \mathrm{CDCl}_{3}\right) \square 7.52-7.58$ (m, 4H, Ar-H), 7.47 (tt, J= 7.3, 1.3 Hz, 1H, Ar-H), 7.36-7.42 (m, 4H, Ar-H), 7.29 (t, $J=7.3 \mathrm{~Hz}, 1 \mathrm{H}, \mathrm{Ar}-\mathbf{H}), 7.01$ (d, $J=6.1 \mathrm{~Hz}, 1 \mathrm{H}, \mathrm{PhCON}-\mathbf{H}), 5.86$ (ddt, $J$ $\left.=17.0,10.0,7.2 \mathrm{~Hz}, 1 \mathrm{H}, \mathrm{CH}_{2} \mathrm{CH}=\mathrm{CH}_{2}\right), 5.56(\mathrm{~d}, J=7.3 \mathrm{~Hz}, 1 \mathrm{H}, \operatorname{ArC}-\mathrm{NH}), 5.09-5.17(\mathrm{~m}, 2 \mathrm{H}$, 
$\left.\mathrm{CH}_{2} \mathrm{CH}=\mathrm{CH}_{2}\right), 2.62-2.71\left(\mathrm{~m}, 2 \mathrm{H}, \mathrm{CH}_{2} \mathrm{CH}=\mathrm{CH}_{2}\right), 1.79-1.93\left(\mathrm{~m}, 2 \mathrm{H}, \mathrm{CH}_{2}-\mathrm{CH}_{3}\right), 0.83\left(\mathrm{~s}, 3 \mathrm{H}, \mathrm{CH}_{2}-\mathrm{CH}_{3}\right)$; ${ }^{13} \mathrm{C}$ NMR $\left(100 \mathrm{MHz}, \mathrm{CDCl}_{3}\right) \square 165.4,142.7,133.9,132.7,131.3,128.4,128.2,126.8,126.51,126.47$, 117.8, 65.0, 40.4, 29.3, 8.0; IR (thin film) 3279, 3058, 2974, 2933, 1638, 1578, 1538, 1447, 1432, 1312 , $916 \mathrm{~cm}^{-1}$; LRMS (FAB+) calcd for $\mathrm{C}_{19} \mathrm{H}_{22} \mathrm{~N}_{2} \mathrm{O} 294$, found $(\mathrm{M}+\mathrm{H})^{+} 295$.

(S)-Benzoic acid $N^{\prime}$-(1-benzyl-1-phenyl-but-3-enyl)-hydrazide (Table 1, Entry 3): $[\square]_{\mathrm{D}}=+0.90^{\circ}$ $\left(c\right.$ 1.4, $\left.\mathrm{CHCl}_{3}\right) ;{ }^{1} \mathrm{H} \mathrm{NMR}\left(400 \mathrm{MHz}, \mathrm{CDCl}_{3}\right) \square 7.50$ (d, J= 7.2 Hz, 2H, Ar-H), 7.41-7.46 (m, 3H, Ar-H), 7.32-7.37 (m, 4H, Ar-H), 7.24-7.29 (m, 1H, Ar-H), 7.10-7.18 (m, 4H, Ar-H), 6.84-6.87 (m, 2H, Ar-H and PhCON-H), 5.97-6.08 (m, 1H, $\left.\mathrm{CH}_{2} \mathrm{CH}=\mathrm{CH}_{2}\right), 5.67(\mathrm{~d}, J=7.9 \mathrm{~Hz}, 1 \mathrm{H}, \operatorname{ArC}-\mathrm{NH})$, 5.15-5.22 (m, 2H, $\left.\mathrm{CH}_{2} \mathrm{CH}=\mathrm{CH}_{2}\right), 3.12\left(\mathrm{~s}, 2 \mathrm{H}, \mathrm{CH}_{2} \mathrm{Ph}\right), 2.80\left(\mathrm{dd}, J=14.8,6.2 \mathrm{~Hz}, 1 \mathrm{H}\right.$, one of $\left.\mathrm{CH}_{2} \mathrm{CH}=\mathrm{CH}_{2}\right), 2.73(\mathrm{dd}, J=$ 14.8, $7.7 \mathrm{~Hz}, 1 \mathrm{H}$, one of $\left.\mathrm{CH}_{2} \mathrm{CH}=\mathrm{CH}_{2}\right) ;{ }^{13} \mathrm{C} \mathrm{NMR}\left(100 \mathrm{MHz}, \mathrm{CDCl}_{3}\right) \square 165.2,142.1,136.2,134.1,132.6$, 131.2, 130.3, 128.3, 128.1, 127.6, 126.9, 126.8, 126.4, 126.3, 118.3, 65.6, 45.7, 39.9; IR (thin film) 3276, 3060, 3027, 2929, 1635, 1576, 1540, 1496, 1447, 1436, 1312, 1026, 920, $765 \mathrm{~cm}^{-1}$; LRMS (FAB+) calcd for $\mathrm{C}_{24} \mathrm{H}_{24} \mathrm{~N}_{2} \mathrm{O} 356$, found $(\mathrm{M}+\mathrm{H})^{+} 357$.

(S)-Benzoic acid $N^{\prime}$-(1-isopropyl-1-phenyl-but-3-enyl)-hydrazide (Table 1, Entry 4): $[\square]_{\mathrm{D}}=$ $+46.9^{\circ}\left(c\right.$ 1.0, $\left.\mathrm{CHCl}_{3}\right) ;{ }^{1} \mathrm{H} \mathrm{NMR}\left(400 \mathrm{MHz} \mathrm{CDCl}_{3}\right) \square 7.56-7.58$ (m, 2H, Ar-H), 7.51-7.53 (m, 2H, Ar-H), 7.43-7.47 (m, 1H, Ar-H), 7.35-7.41 (m, 4H, Ar-H), 7.29 (t, $J=7.3 \mathrm{~Hz}, 1 \mathrm{H}$, Ar-H), $7.21(\mathrm{~d}, J=8.0 \mathrm{~Hz}$, 1H, PhCON-H), 5.99-6.09 (m, 1H, $\left.\mathrm{CH}_{2} \mathrm{CH}=\mathrm{CH}_{2}\right), 5.67(\mathrm{~d}, J=8.4 \mathrm{~Hz}, 1 \mathrm{H}, \operatorname{ArC}-\mathrm{NH}), 5.20(\mathrm{dd}, J=17.0$, $1.3 \mathrm{~Hz}, 1 \mathrm{H}$, one of $\left.\mathrm{CH}_{2} \mathrm{CH}=\mathrm{CH}_{2}\right), 5.10\left(\mathrm{~d}, J=10 \mathrm{~Hz}, 1 \mathrm{H}\right.$, one of $\left.\mathrm{CH}_{2} \mathrm{CH}=\mathrm{CH}_{2}\right), 2.80-2.90(\mathrm{~m}, 2 \mathrm{H}$, $\left.\mathrm{CH}_{2} \mathrm{CH}=\mathrm{CH}_{2}\right), 2.03\left(\mathrm{sep}, J=6.8 \mathrm{~Hz}, 1 \mathrm{H}, \mathrm{H}-\mathrm{C}\left(\mathrm{CH}_{3}\right)_{2}\right), 0.89\left(\mathrm{~d}, J=6.8 \mathrm{~Hz}, 3 \mathrm{H}\right.$, one of CH- $\left.\left(\mathrm{CH}_{3}\right)_{2}\right), 0.85$ $\left(\mathrm{d}, J=6.8 \mathrm{~Hz}, 3 \mathrm{H}\right.$, one of $\left.\mathrm{CH}-\left(\mathrm{CH}_{3}\right)_{2}\right) ;{ }^{13} \mathrm{C} \mathrm{NMR}\left(100 \mathrm{MHz}, \mathrm{CDCl}_{3}\right) \square 164.9,140.3,134.7,132.8,131.3$, $128.4,127.9,127.5,126.6,126.4,117.6,67.8,38.6,35.3,17.8,17.4$; IR (thin film) 3284, 3065, 2963, 2876, 1637, 1579, 1535, 1492, 1447, 1427, 1307, $918 \mathrm{~cm}^{-1}$; LRMS (FAB+) calcd for $\mathrm{C}_{20} \mathrm{H}_{24} \mathrm{~N}_{2} \mathrm{O} 308$, found $(\mathrm{M}+\mathrm{H})^{+} 309$.

(R)- 2-(N'-Benzoyl-hydrazino)-2-phenyl-pent-4-enoic acid methyl ester (Table 1, Entry 5): $[\square]_{\mathrm{D}}=$ $-36.0^{\circ}$ (c 1.15, CHCl3); ${ }^{1} \mathrm{H}$ NMR (400 $\left.\mathrm{MHz}, \mathrm{CDCl}_{3}\right) \square 7.80(\mathrm{~d}, J=6.4 \mathrm{~Hz}, 1 \mathrm{H}, \mathrm{PhCON}-\mathbf{H}), 7.55-7.58(\mathrm{~m}$, 2H, Ar-H), 7.44-7.48 (m, 3H, Ar-H), 7.27-7.39 (m, 5H, Ar-H), 5.82-5.92 (m, 1H, $\left.\mathrm{CH}_{2} \mathrm{CH}^{-} \mathrm{CH}_{2}\right), 5.65(\mathrm{~d}$, $J=6.7 \mathrm{~Hz}, 1 \mathrm{H}, \mathrm{ArC}-\mathrm{NH}), 5.20\left(\mathrm{~d}, J=17.0 \mathrm{~Hz}, 1 \mathrm{H}\right.$, one of $\left.\mathrm{CH}_{2} \mathrm{CH}=\mathrm{CH}_{2}\right), 5.15(\mathrm{~d}, J=10.0 \mathrm{~Hz}, 1 \mathrm{H}$, one 
of $\left.\mathrm{CH}_{2} \mathrm{CH}=\mathrm{CH}_{2}\right), 3.80\left(\mathrm{~s}, 3 \mathrm{H}, \mathrm{CO}_{2} \mathrm{CH}_{3}\right), 3.01\left(\mathrm{dd}, J=14.3,7.4 \mathrm{~Hz}, 1 \mathrm{H}\right.$, one of $\left.\mathrm{CH}_{2} \mathrm{CH}=\mathrm{CH}_{2}\right), 2.94(\mathrm{dd}, J$ $=14.3,6.9 \mathrm{~Hz}, 1 \mathrm{H}$, one of $\left.\mathrm{CH}_{2} \mathrm{CH}=\mathrm{CH}_{2}\right) ;{ }^{13} \mathrm{C} \mathrm{NMR}\left(100 \mathrm{MHz}, \mathrm{CDCl}_{3}\right) \square 173.4,165.8,138.4,132.6$, $132.4,131.3,128.3,128.1,127.9,126.5,126.4,119.2,71.0,52.5,41.0$; IR (thin film) $3280,3063,3020$, $2951,1733,1642,1602,1579,1532,1495,1448,1433,1231,698 \mathrm{~cm}^{-1}$; LRMS (FAB+) calcd for $\mathrm{C}_{19} \mathrm{H}_{20} \mathrm{~N}_{2} \mathrm{O}_{3} 324$, found $(\mathrm{M}+\mathrm{H})^{+} 325$.

R)-Benzoic acid $N^{\prime}$-(1-(4-bromophenyl)-1-methyl-but-3-enyl)-hydrazide (Table 1, Entry 6): $[\square]_{\mathrm{D}}=+91.8^{\circ}\left(c 1.0, \mathrm{CHCl}_{3}\right) ;{ }^{1} \mathrm{H} \mathrm{NMR}\left(400 \mathrm{MHz} \mathrm{CDCl}_{3}\right) \square 7.59(\mathrm{~d}, J=7.8 \mathrm{~Hz}, 2 \mathrm{H}, \mathrm{Ar}-\mathbf{H}), 7.37-7.51(\mathrm{~m}$, 7H, Ar-H), 7.09 (br s, 1H, PhCON-H), 5.58-5.67 (m, 1H, $\mathrm{CH}_{2} \mathrm{CH}=\mathrm{CH}_{2}$ ), 5.41 (br s, 1H, ArC-NH), 5.10 $\left(\mathrm{d}, J=3.8 \mathrm{~Hz}, 1 \mathrm{H}\right.$, one of $\left.\mathrm{CH}_{2} \mathrm{CH}=\mathrm{CH}_{2}\right), 5.07\left(\mathrm{~s}, 1 \mathrm{H}\right.$, one of $\left.\mathrm{CH}_{2} \mathrm{CH}=\mathrm{CH}_{2}\right), 2.57(\mathrm{dd}, J=13.7,6.6 \mathrm{~Hz}$, $1 \mathrm{H}$, one of $\left.\mathrm{CH}_{2} \mathrm{CH}=\mathrm{CH}_{2}\right), 2.47\left(\mathrm{dd}, J=13.7,8.0 \mathrm{~Hz}, 1 \mathrm{H}\right.$, one of $\left.\mathrm{CH}_{2} \mathrm{CH}=\mathrm{CH}_{2}\right), 1.53\left(\mathrm{~s}, 3 \mathrm{H}, \mathrm{ArC}-\mathrm{CH}_{3}\right)$; ${ }^{13} \mathrm{C}$ NMR $\left(100 \mathrm{MHz}, \mathrm{CDCl}_{3}\right) \square 166.5,143.3,132.8,132.4,131.5,131.3,128.4,128.2,126.5,120.9$, 118.8, 62.3, 46.1, 22.7; IR (thin film) 3274, 3071, 2977, 1638, 1578, 1540, 1487, 1449, 1395, 1314, 1008 $\mathrm{cm}^{-1}$; LRMS (FAB+) calcd for $\mathrm{C}_{18} \mathrm{H}_{19} \mathrm{BrN}_{2} \mathrm{O} 358$, found $(\mathrm{M}+\mathrm{H})^{+} 359$.

(R)-Benzoic acid $N^{\prime}$-(1-(4-methoxy-phenyl)-1-methyl-but-3-enyl)-hydrazide (Table 1, Entry 7): $[\square]_{\mathrm{D}}=+86.6^{\circ}\left(c 1.8, \mathrm{CHCl}_{3}\right) ;{ }^{1} \mathrm{H} \mathrm{NMR}\left(400 \mathrm{MHz} \mathrm{CDCl}_{3}\right) \square 7.58-7.60(\mathrm{~m}, 2 \mathrm{H}, \mathrm{Ar}-\mathbf{H})$, 7.44-7.47 (m, 3H, $\operatorname{Ar}-\mathbf{H})$, 7.35-7.38 (m, 2H, Ar-H), 7.14 (br s, 1H, PhCON-H), 6.91 (d, J=8.8 Hz, 2H, Ar-H), 5.61-5.72 (m, $1 \mathrm{H}, \mathrm{CH}_{2} \mathrm{CH}=\mathrm{CH}_{2}$ ), 5.39 (br s, $\left.1 \mathrm{H}, \mathrm{ArC}-\mathrm{NH}\right), 5.05-5.10\left(\mathrm{~m}, 2 \mathrm{H}, \mathrm{CH}_{2} \mathrm{CH}=\mathrm{CH}_{2}\right), 3.81\left(\mathrm{~s}, 3 \mathrm{H}, \mathrm{OCH}_{3}\right)$, $2.60\left(\mathrm{dd}, J=13.7,6.6 \mathrm{~Hz}, 1 \mathrm{H}\right.$, one of $\left.\mathrm{CH}_{2} \mathrm{CH}=\mathrm{CH}_{2}\right), 2.49(\mathrm{dd}, J=13.7,8.0 \mathrm{~Hz}, 1 \mathrm{H}$, one of $\left.\mathrm{CH}_{2} \mathrm{CH}=\mathrm{CH}_{2}\right), 1.52\left(\mathrm{~s}, 3 \mathrm{H}, \operatorname{ArC}-\mathrm{CH}_{3}\right) ;{ }^{13} \mathrm{C} \mathrm{NMR}\left(100 \mathrm{MHz}, \mathrm{CDCl}_{3}\right) \square 166.1,158.1,135.8,133.4,132.6$, 131.3, 128.3, 127.4, 126.5, 118.2, 113.5, 62.0, 55.2, 46.1, 22.8; IR (thin film) 3276, 3070, 2975, 2933, $2835,1638,1610,1512,1451,1440,1301,1250,1181,1033 \mathrm{~cm}^{-1}$; LRMS (FAB+) calcd for $\mathrm{C}_{19} \mathrm{H}_{22} \mathrm{~N}_{2} \mathrm{O}$ 310 , found $(\mathrm{M}+\mathrm{H})^{+} 311$.

(R)-Benzoic acid $N^{\prime}$-(1-methyl-1-(3-nitrophenyl)-but-3-enyl)-hydrazide (Table 1, Entry 8): $[\square]_{\mathrm{D}}=$ $+64.9^{\circ}\left(c\right.$ 2.2, $\left.\mathrm{CHCl}_{3}\right) ;{ }^{1} \mathrm{H} \mathrm{NMR}\left(400 \mathrm{MHz}, \mathrm{CDCl}_{3}\right) \square 8.42(\mathrm{t}, J=2.0 \mathrm{~Hz}, 1 \mathrm{H}, \mathrm{Ar}-\mathbf{H}), 8.10-8.13(\mathrm{~m}, 1 \mathrm{H}$, $\operatorname{Ar}-\mathbf{H}), 7.90(\mathrm{~d}, J=7.9 \mathrm{~Hz}, 1 \mathrm{H}, \operatorname{Ar}-\mathbf{H}), 7.59-7.62(\mathrm{~m}, 2 \mathrm{H}, \mathrm{Ar}-\mathbf{H}), 7.53$ (t, $J=8.0 \mathrm{~Hz}, 1 \mathrm{H}, \operatorname{Ar}-\mathbf{H})$, 7.45-7.49 $(\mathrm{m}, 1 \mathrm{H}, \operatorname{Ar}-\mathbf{H}), 7.35-7.39$ (m, 2H, Ar-H), 7.31 (d, J = 7.5 Hz, 1H, PhCON-H), 5.57-5.68 (m, 1H, $\left.\mathrm{CH}_{2} \mathrm{CH}=\mathrm{CH}_{2}\right), 5.46(\mathrm{~d}, J=7.8 \mathrm{~Hz}, 1 \mathrm{H}, \mathrm{ArC}-\mathrm{NH}), 5.06-5.11\left(\mathrm{~m}, 2 \mathrm{H}, \mathrm{CH}_{2} \mathrm{CH}=\mathrm{CH}_{2}\right), 2.61(\mathrm{dd}, J=13.8$, 
6.7 Hz, $1 \mathrm{H}$, one of $\left.\mathrm{CH}_{2} \mathrm{CH}=\mathrm{CH}_{2}\right), 2.52\left(\mathrm{dd}, J=13.8,7.9 \mathrm{~Hz}, 1 \mathrm{H}\right.$, one of $\left.\mathrm{CH}_{2} \mathrm{CH}=\mathrm{CH}_{2}\right), 1.61(\mathrm{~s}, 3 \mathrm{H}, \mathrm{ArC}-$ $\left.\mathrm{CH}_{3}\right) ;{ }^{13} \mathrm{C}$ NMR $\left(100 \mathrm{MHz}, \mathrm{CDCl}_{3}\right) \square 167.0,147.9,146.6,132.7,132.2,132.0,131.5,128.9,128.3,126.6$, 121.8, 121.5, 119.1, 62.4, 45.8, 22.5, ; IR (thin film) 3275, 3083, 2981, 1627, 1448, 1348, 1312, 918, 865, $694 \mathrm{~cm}^{-1}$; LRMS (FAB+) calcd for $\mathrm{C}_{18} \mathrm{H}_{19} \mathrm{~N}_{3} \mathrm{O}_{3} 325$, found $(\mathrm{M}+\mathrm{H})^{+} 326$.

(R)-Benzoic acid $N^{\prime}$-(1-methyl-1-napthalen-2-yl-but-3-enyl)-hydrazide (Table 1, Entry 9): $[\square]_{\mathrm{D}}=$ $+105.3^{\circ}\left(c\right.$ 1.25, $\left.\mathrm{CHCl}_{3}\right) ;{ }^{1} \mathrm{H}$ NMR (400 $\left.\mathrm{MHz} \mathrm{CDCl}_{3}\right) \square 7.84-7.93$ (m, 4H, Ar-H), 7.77 (dd, J = 8.6, 1.8 Hz, 1H, Ar-H), 7.42-7.57 (m, 5H, Ar-H), 7.34 (t, J= 7.5 Hz, 2H, Ar-H), 7.11 (br s, 1H, PhCON-H), 5.63$5.73\left(\mathrm{~m}, 1 \mathrm{H}, \mathrm{CH}_{2} \mathrm{CH}=\mathrm{CH}_{2}\right), 5.55$ (br s, $\left.1 \mathrm{H}, \mathrm{ArC}-\mathrm{NH}\right), 5.12\left(\mathrm{~d}, J=17.1 \mathrm{~Hz}, 1 \mathrm{H}\right.$, one of $\left.\mathrm{CH}_{2} \mathrm{CH}_{=} \mathrm{CH}_{2}\right)$, $5.08\left(\mathrm{~d}, J=10.1 \mathrm{~Hz}, 1 \mathrm{H}\right.$, one of $\left.\mathrm{CH}_{2} \mathrm{CH}=\mathrm{CH}_{2}\right), 2.72\left(\mathrm{dd}, J=13.6,6.6 \mathrm{~Hz}, 1 \mathrm{H}\right.$, one of $\left.\mathrm{CH}_{2} \mathrm{CH}_{=} \mathrm{CH}_{2}\right), 2.60$ $\left(\mathrm{dd}, J=13.6,8.0 \mathrm{~Hz}, 1 \mathrm{H}\right.$, one of $\left.\mathrm{CH}_{2} \mathrm{CH}=\mathrm{CH}_{2}\right), 1.67\left(\mathrm{~s}, 3 \mathrm{H}, \operatorname{ArC}-\mathrm{CH}_{3}\right) ;{ }^{13} \mathrm{C} \mathrm{NMR}\left(100 \mathrm{MHz}, \mathrm{CDCl}_{3}\right)$ $\square 166.3,141.5,133.2,133.0,132.5,132.2,131.4,128.4,128.1,128.0,127.3,126.5,126.0,125.8,125.3$, 124.5, 118.6, 62.6, 46.1, 22.8; IR (thin film) 3275, 3060, 2976, 1638, 1578, 1539, 1449, 1436, 1375, 1312. $915 \mathrm{~cm}^{-1}$; LRMS (FAB+) calcd for $\mathrm{C}_{22} \mathrm{H}_{22} \mathrm{~N}_{2} \mathrm{O} 330$, found $(\mathrm{M}+\mathrm{H})^{+} 331$.

(R)-Benzoic acid $N^{\prime}$-(1-furan-2-yl-1-methyl-but-3-enyl)-hydrazide (Table 1, Entry 10): $[\square]_{\mathrm{D}}=$ $+37.9^{\circ}\left(c\right.$ 1.5, $\left.\mathrm{CHCl}_{3}\right) ;{ }^{1} \mathrm{H} \mathrm{NMR}\left(400 \mathrm{MHz} \mathrm{CDCl}_{3}\right) \square 7.64-7.67(\mathrm{~m}, 2 \mathrm{H}, \mathrm{Ar}-\mathbf{H}), 7.49$ (tt, J = 7.4, $1.2 \mathrm{~Hz}$, 1H, Ar-H), 7.39-7.43 (m, 3H, Ar-H), 7.29 (br s, 1H, PhCON-H), 6.32 (dd, J = 3.2, 1.8 Hz, 1H, Ar-H), $6.23(\mathrm{dd}, J=3.2,0.5 \mathrm{~Hz}, 1 \mathrm{H}, \mathrm{Ar}-\mathbf{H}), 5.73\left(\mathrm{ddt}, J=17.0,10.0,7.6 \mathrm{~Hz}, 1 \mathrm{H}, \mathrm{CH}_{2} \mathrm{CH}=\mathrm{CH}_{2}\right), 5.24(\mathrm{br}$ s, $1 \mathrm{H}$, ArC-NH), 5.08-5.15 (m, 2H, $\left.\mathrm{CH}_{2} \mathrm{CH}=\mathrm{CH}_{2}\right), 2.73\left(\mathrm{dd}, J=13.7,6.7 \mathrm{~Hz}, 1 \mathrm{H}\right.$, one of $\left.\mathrm{CH}_{2} \mathrm{CH}=\mathrm{CH}_{2}\right), 2.55$ $\left(\mathrm{dd}, J=13.7,7.8 \mathrm{~Hz}, 1 \mathrm{H}\right.$, one of $\left.\mathrm{CH}_{2} \mathrm{CH}=\mathrm{CH}_{2}\right), 1.48\left(\mathrm{~s}, 3 \mathrm{H}, \operatorname{ArC}-\mathrm{CH}_{3}\right) ;{ }^{13} \mathrm{C} \mathrm{NMR}\left(100 \mathrm{MHz}, \mathrm{CDCl}_{3}\right)$ 166.5, 156.5, 141.7, 133.0, 132.6, 131.4, 128.3, 126.6, 118.3, 109.9, 106.7, 59.8, 42.5, 21.5; IR (thin film) $3271,3067,2980,1640,1576,1542,1456,1436,1379,1314,1160,1014 \mathrm{~cm}^{-1}$; LRMS (FAB+) calcd for $\mathrm{C}_{16} \mathrm{H}_{18} \mathrm{~N}_{2} \mathrm{O}_{2} 270$, found $(\mathrm{M}+\mathrm{H})^{+} 271$.

(R)-Benzoic acid $N^{\prime}$-(1-methyl-1-thiophen-2-yl-but-3-enyl)-hydrazide (Table 1, Entry 11): $[\square]_{\mathrm{D}}=$ $+49.6^{\circ}\left(c\right.$ 2.0, $\left.\mathrm{CHCl}_{3}\right) ;{ }^{1} \mathrm{H} \mathrm{NMR}\left(400 \mathrm{MHz}, \mathrm{CDCl}_{3}\right) \square 7.64-7.66(\mathrm{~m}, 2 \mathrm{H}, \mathrm{Ar}-\mathbf{H}), 7.49$ (t, J = 7.4 Hz, 1H, Ar-H), 7.39-7.42 (m, 2H, Ar-H), 7.25-7.28 (m, 2H, PhCON-H and Ar-H), 6.96-7.00 (m, 2H, Ar-H), 5.78 (ddt, $J=17.0,10.0,7.4 \mathrm{~Hz}, 1 \mathrm{H}, \mathrm{CH}_{2} \mathrm{CH}=\mathrm{CH}_{2}$ ), 5.43 (br s, $\left.1 \mathrm{H}, \operatorname{ArC}-\mathrm{NH}\right), 5.12-5.16(\mathrm{~m}, 2 \mathrm{H}$, $\left.\mathrm{CH}_{2} \mathrm{CH}=\mathrm{CH}_{2}\right), 2.66\left(\mathrm{dd}, J=13.7,6.7 \mathrm{~Hz}, 1 \mathrm{H}\right.$, one of $\left.\mathrm{CH}_{2} \mathrm{CH}=\mathrm{CH}_{2}\right), 2.59(\mathrm{dd}, J=13.7,7.9 \mathrm{~Hz}, 1 \mathrm{H}$, one 
of $\left.\mathrm{CH}_{2} \mathrm{CH}=\mathrm{CH}_{2}\right), 1.58$ (s, 3H, ArC-CH$\left.) ;{ }^{13} \mathrm{C} \mathrm{NMR} \mathrm{(100} \mathrm{MHz,} \mathrm{CDCl}_{3}\right) \square$ 166.5, 149.5, 132.9, 132.4, $131.5,128.3,126.6,126.5,124.5,124.1,118.6,61.8,46.5,23.8$; IR (thin film) 3273, 3071, 2977, 2932 , $1638,1587,1540,1450,1435,1372,1312,917 \mathrm{~cm}^{-1}$; LRMS (FAB+) calcd for $\mathrm{C}_{16} \mathrm{H}_{18} \mathrm{~N}_{2} \mathrm{OS} 286$, found $(\mathrm{M}+\mathrm{H})^{+} 287$.

(R)-3-[1-( $N^{\prime}$-Benzoyl-hydrazino)-1-methyl-but-3-enyl]-indole-1-carboxylic acid $t$-butyl ester (Table 1, Entry 12): $[\square]_{\mathrm{D}}=+51.3^{\circ}\left(\mathrm{c} 1.24, \mathrm{CHCl}_{3}\right) ;{ }^{1} \mathrm{H} \mathrm{NMR}\left(400 \mathrm{MHz}, \mathrm{CDCl}_{3}\right) \square 8.20(\mathrm{~s}, 1 \mathrm{H}, \mathrm{Ar}-\mathrm{H})$, $8.16(\mathrm{~d}, J=8.0 \mathrm{~Hz}, 1 \mathrm{H}, \mathrm{Ar}-\mathbf{H}), 7.57-7.59$ (m, 2H, Ar-H), 7.43-7.47 (m, 2H, Ar-H), 7.33-7.38 (m, 3H, ArH), 7.24-7.28 (m, 1H, Ar-H), 7.04 (br s, 1H, PhCON-H), 5.70-5.81 (m, 1H, $\left.\mathrm{CH}_{2} \mathrm{CH}=\mathrm{CH}_{2}\right), 5.48$ (br s, $1 \mathrm{H}$, ArC-NH), 5.07-5.13 (m, 2H, $\left.\mathrm{CH}_{2} \mathrm{CH}=\mathrm{CH}_{2}\right), 2.82\left(\mathrm{dd}, J=13.5,6.5 \mathrm{~Hz}, 1 \mathrm{H}\right.$, one of $\left.\mathrm{CH}_{2} \mathrm{CH}_{=} \mathrm{CH}_{2}\right), 2.66$ $\left(\mathrm{dd}, J=13.5,8.0 \mathrm{~Hz}, 1 \mathrm{H}\right.$, one of $\left.\mathrm{CH}_{2} \mathrm{CH}=\mathrm{CH}_{2}\right), 1.69\left(\mathrm{~s}, 9 \mathrm{H}, \mathrm{COC}\left(\mathrm{CH}_{3}\right)_{3}\right), 1.57\left(\mathrm{~s}, 3 \mathrm{H}, \operatorname{ArC}-\mathrm{CH}_{3}\right) ;{ }^{13} \mathrm{C}$ NMR (100 MHz, $\left.\mathrm{CDCl}_{3}\right) \square 166.3,149.4,135.9,133.2,132.4,131.4,128.3,126.5,124.2,123.8,123.6$, $122.4,121.8,118.5,115.1,83.8,60.6,43.7,28.3,23.0$; IR (thin film) 3242, 3052, 2979, 2931, 1734, 1654, 1559, 1451, 1370, 1309, 1281, 1243, 1153, 1066, $750 \mathrm{~cm}^{-1}$; LRMS (FAB+) calcd for $\mathrm{C}_{25} \mathrm{H}_{29} \mathrm{~N}_{3} \mathrm{O}_{3}$ 419, found $(\mathrm{M}+\mathrm{H})^{+} 420$.

(R)-Benzoic acid $N^{\prime}$-(1-methyl-1-phenethyl-but-3-enyl)-hydrazide (Table 1, Entry 13): $[\square]_{\mathrm{D}}=$ $+10.8^{\circ}\left(c\right.$ 2.7, $\left.\mathrm{CHCl}_{3}\right) ;{ }^{1} \mathrm{H} \mathrm{NMR}\left(400 \mathrm{MHz} \mathrm{CDCl}_{3}\right) \square 7.69-7.71(\mathrm{~m}, 2 \mathrm{H}, \mathrm{Ar}-\mathbf{H}), 7.50$ (tt, J = 7.3, $1.3 \mathrm{~Hz}$, 1H, Ar-H), 7.41-7.45 (m, 2H, Ar-H), 7.15-7.29 (m, 6H, PhCON-H and Ar-H), 5.97 (ddt, J = 17.0, 10.0, $\left.7.3 \mathrm{~Hz}, 1 \mathrm{H}, \mathrm{CH}_{2} \mathrm{CH}=\mathrm{CH}_{2}\right), 5.14-5.20\left(\mathrm{~m}, 2 \mathrm{H}, \mathrm{CH}_{2} \mathrm{CH}=\mathrm{CH}_{2}\right), 5.08$ (br s, $\left.1 \mathrm{H}, \operatorname{ArC}-\mathrm{NH}\right), 2.74(\mathrm{t}, J=8.5$ $\left.\mathrm{Hz}, 2 \mathrm{H}, \mathrm{PhCH}_{2} \mathrm{CH}_{2}\right), 2.26-2.36\left(\mathrm{~m}, 2 \mathrm{H}, \mathrm{CH}_{2} \mathrm{CH}=\mathrm{CH}_{2}\right), 1.70-1.82\left(\mathrm{~m}, 2 \mathrm{H}, \mathrm{PhCH}_{2} \mathrm{CH}_{2}\right), 1.18(\mathrm{~s}, 3 \mathrm{H}, \mathrm{ArC}-$ $\left.\mathrm{CH}_{3}\right) ;{ }^{13} \mathrm{C}$ NMR $\left(100 \mathrm{MHz}, \mathrm{CDCl}_{3}\right) \square 166.8,142.3,134.1,132.6,131.4,128.3,128.1,126.6,125.5,117.8$, 59.5, 42.7, 39.7, 30.1, 23.0; IR (thin film) 3279, 3062, 3025, 2973, 2932, 2849, 1635, 1456, 1436, 1312 , 913, $690 \mathrm{~cm}^{-1}$; LRMS (FAB+) calcd for $\mathrm{C}_{20} \mathrm{H}_{24} \mathrm{~N}_{2} \mathrm{O} 308$, found $(\mathrm{M}+\mathrm{H})^{+} 309$.

(R)-Benzoic acid $N^{\prime}$-(1-cyclohexyl-1-methyl-but-3-enyl)-hydrazide (Table 1, Entry 14): $[\square]_{\mathrm{D}}=$ 9.78 $\left(c\right.$ 1.25, $\left.\mathrm{CHCl}_{3}\right) ;{ }^{1} \mathrm{H} \mathrm{NMR}\left(400 \mathrm{MHz}, \mathrm{CDCl}_{3}\right) \square 7.70-7.73$ (m, 2H, Ar-H), 7.41-7.52 (m, 3H, Ar-H), 7.26 (br s, 1H, PhCON-H), 6.02 (ddt, $J=17.0,10.0,7.1 \mathrm{~Hz}, 1 \mathrm{H}, \mathrm{CH}_{2} \mathrm{CH}=\mathrm{CH}_{2}$ ), 5.12-5.17 (m, 3H, $\mathrm{CH}_{2} \mathrm{CH}=\mathrm{CH}_{2}$ and $\left.\mathrm{ArC}-\mathrm{NH}\right), 2.21-2.32\left(\mathrm{~m}, 2 \mathrm{H}, \mathrm{CH}_{2} \mathrm{CH}=\mathrm{CH}_{2}\right), 1.93(\mathrm{~d}, J=1.8 \mathrm{~Hz}, 1 \mathrm{H}, \mathrm{Cy}-\mathbf{H}), 1.79-1.87$ (m, 3H, Cy-H), 1.68 (d, J = 1.4 Hz, 1H, Cy-H), 1.39-1.45 (m, 1H, Cy-H), 1.06-1.27 (m, 5H, Cy-H), 1.05 
(s, 3H, ArC-CH $\left.{ }_{3}\right) ;{ }^{13} \mathrm{C}$ NMR (100 MHz, $\left.\mathrm{CDCl}_{3}\right) \square 166.4,135.0,132.8,131.3,128.3,126.5,117.1,61.6$, 44.2, 40.9, 27.4, 27.1, 27.04, 26.99, 26.7, 20.2 ; IR (thin film) 3287, 3070, 2926, 2851, 1635, 1549, 1450, $1375,1313,1159 \mathrm{~cm}^{-1}$; LRMS (FAB+) calcd for $\mathrm{C}_{18} \mathrm{H}_{26} \mathrm{~N}_{2} \mathrm{O} 286$, found $(\mathrm{M}+\mathrm{H})^{+} 287$.

\section{Procedure for the enantioselective allylation of benzoic acid (1-phenyl-ethylidene)-hydrazide} with $(S, S)-1$ on a $5.0 \mathrm{~g}$ scale (Scheme 2): To a solution of $(S, S)-\mathbf{1}(8.43 \mathrm{~g}, 31.5 \mathrm{mmol})$ in $\mathrm{CHCl}_{3}(210$ $\mathrm{mL}$ ) was added benzoic acid (1-phenyl-ethylidene)-hydrazide $(5.00 \mathrm{~g}, 21.0 \mathrm{mmol})$. The resulting solution was heated at $40{ }^{\circ} \mathrm{C}$ for 24 hours, then cooled to ambient temperature and quenched with methanol (40 $\mathrm{mL})$. After $15 \mathrm{~min}$, the solution was concentrated and the residue diluted with EtOAc $(100 \mathrm{~mL})$ and $\mathrm{H}_{2} \mathrm{O}$ $(200 \mathrm{~mL})$. The phases were separated, and the aqueous layer was extracted with EtOAc $(2 \times 100 \mathrm{~mL})$. The combined organic layers were washed $\left(2 \times 100 \mathrm{~mL} \mathrm{H}_{2} \mathrm{O}, 1 \times 100 \mathrm{~mL}\right.$ brine $)$, dried $\left(\mathrm{MgSO}_{4}\right)$, filtered and

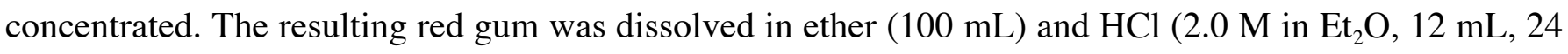
mmol) was added. A white precipitate formed immediately, and after 10 min was filtered and washed with ether $(2 \times 20 \mathrm{~mL})$. The off-white solid was dried under vacuum to yield the crude product as the $\mathrm{HCl}$ salt. Recrystallization from absolute ethanol yielded the pure product $(R)$-Benzoic acid $N^{\prime}$-(1-methyl-1phenyl-but-3-enyl)-hydrazide- $\mathrm{HCl}(4.90 \mathrm{~g}, 74 \%)$ as a crystalline white solid. (The pseudoephedrine was recovered by the following procedure: the combined aqueous extracts from the above workup were basified with $3 \mathrm{~N}$ aqueous $\mathrm{NaOH}$ and the cloudy suspension was extracted with EtOAc $(3 \times 75 \mathrm{~mL})$. The combined organic layers were washed $\left(2 \times 25 \mathrm{~mL} \mathrm{H}_{2} \mathrm{O}, 1 \times 100 \mathrm{~mL}\right.$ brine), dried $\left(\mathrm{MgSO}_{4}\right)$, filtered and concentrated to yield pure $(S, S)$-pseudoephedrine as a white solid $(5.10 \mathrm{~g}, 98 \%$ recovery $))$. [■] $]_{\mathrm{D}}=-5.5^{\circ}(c$ 1.64, $\mathrm{CD}_{3} \mathrm{OD}$ ); ${ }^{1} \mathrm{H}$ NMR (400 MHz, DMSO- $\left.d_{6}\right) \mathrm{11.1}$ (br s, $\left.1 \mathrm{H}, \mathrm{PhCON}-\mathrm{H}\right), 7.68-7.70$ (m, 2H, Ar-H), 7.53-7.59 (m, 3H, Ar-H), 7.43 (t, $J=7.4 \mathrm{~Hz}, 2 \mathrm{H}, \operatorname{Ar}-\mathbf{H})$, 7.25-7.36 (m, 3H, Ar-H), 5.37-5.47 (m, 1H, $\left.\mathrm{CH}_{2} \mathrm{CH}=\mathrm{CH}_{2}\right), 5.06\left(\mathrm{~d}, J=16 \mathrm{~Hz}, 1 \mathrm{H}\right.$, one of $\left.\mathrm{CH}_{2} \mathrm{CH}=\mathrm{CH}_{2}\right), 4.97-5.00\left(\mathrm{~m}, 1 \mathrm{H}\right.$, one of $\left.\mathrm{CH}_{2} \mathrm{CH}=\mathrm{CH}{ }_{2}\right)$, $3.08\left(\mathrm{dd}, J=13.8,6.0 \mathrm{~Hz}, 1 \mathrm{H}\right.$, one of $\left.\mathrm{CH}_{2} \mathrm{CH}=\mathrm{CH}_{2}\right), 2.73(\mathrm{dd}, J=13.8,8.0 \mathrm{~Hz}, 1 \mathrm{H}$, one of $\left.\mathrm{CH}_{2} \mathrm{CH}=\mathrm{CH}_{2}\right), 1.67$ (s, 3H, ArC-CH 3 ); ${ }^{13} \mathrm{C}$ NMR (100 MHz, $\left.\mathrm{CD}_{3} \mathrm{OD}\right) \square 167.8,136.1,134.1,131.5,131.0$, 130.4, 129.8, 129.6, 128.8, 128.6, 121.0, 70.4, 42.6, 20.6; IR (thin film) 3129, 2949, 2903, 2559, 2489, $2359,1690,1550,1528,1448,1390,1375,1287 \mathrm{~cm}^{-1}$; LRMS (FAB+) calcd for (free base) $\mathrm{C}_{18} \mathrm{H}_{20} \mathrm{~N}_{2} \mathrm{O}$ 280 , found $(\mathrm{M}+\mathrm{H})^{+} 281$. 
Note: A sample of the $\mathrm{HCl}$ salt was free-based and used for the determination of the enantiomeric excess of the recrystallized product. The following procedure was employed: To a stirred solution of the $\mathrm{HCl}$ salt $(25 \mathrm{mg}, 0.08 \mathrm{mmol})$ in $\mathrm{MeOH}$ was added solid $\mathrm{NaOMe}(4.8 \mathrm{mg}, 0.09 \mathrm{mmol})$. After $5 \mathrm{~min}$ the reaction was concentrated and the residue diluted with EtOAc $(2 \mathrm{~mL}) / \mathrm{H}_{2} \mathrm{O}(2 \mathrm{~mL})$. The phases were separated and the aqueous layer extracted with EtOAc $(2 \times 2 \mathrm{~mL})$. The combined organic layers were washed ( $2 \times 2 \mathrm{~mL} \mathrm{H} \mathrm{H}_{2} \mathrm{O}, 1 \times 2 \mathrm{~mL}$ brine), dried $\left(\mathrm{MgSO}_{4}\right)$, filtered and concentrated to yield the product $(R)$-Benzoic acid $N^{\prime}$-(1-methyl-1-phenyl-but-3-enyl)-hydrazide $(21 \mathrm{mg}, 94 \%)$ as the free base. $[\square]_{\mathrm{D}}=$ $+89.9^{\circ}\left(c 0.93, \mathrm{CHCl}_{3}\right)$.

Determination of absolute configuration: $(R)$-Benzoic acid $N^{\prime}$-(1-methyl-1-phenyl-but-3-enyl)hydrazide was reduced to yield the corresponding homoallylic amine (Scheme 1). ${ }^{2}$ Comparison of the optical rotation to the lit. value ${ }^{3}$ established the $(R)$ configuration of our product. All other absolute configurations of the products from Table 1 were assigned by analogy.

\section{Scheme 1}
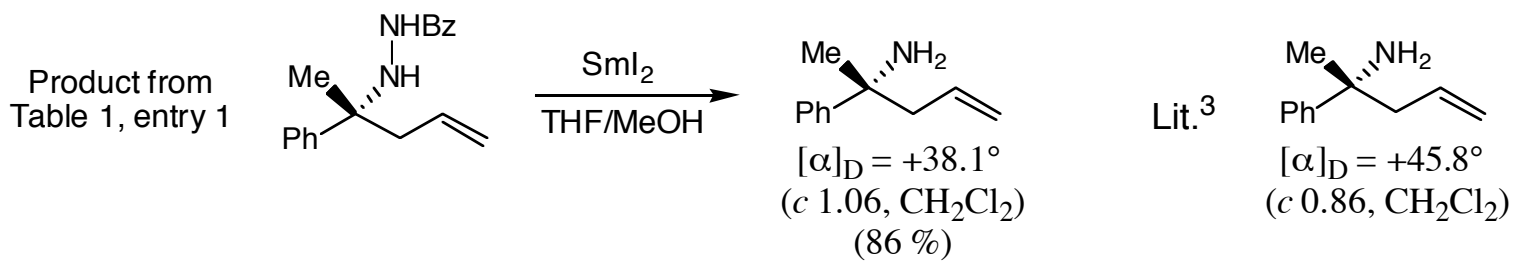

$(86 \%)$

(2) (a) Kobayashi, S.; Hirabayashi, R. J. Am. Chem. Soc. 1999, 121, 6942-6943. (b) Ogawa, C.; Sugiura, M.; Kobayashi, S. J. Org. Chem. 2002, 67, 5359-5364.

(3) Hua, D. H.; Miao, S. W.; Chen, J. S.; Iguchi, S. J. Org. Chem. 1991, 56, 4-6. 
Preparation of reagent $(\boldsymbol{S}, \boldsymbol{S})-7$ : Triethylamine $(27 \mathrm{~mL}, 0.19 \mathrm{~mol})$ was addded to a cooled $\left(0{ }^{\circ} \mathrm{C}\right)$ solution of phenyltrichlorosilane $(15 \mathrm{~mL}, 0.095 \mathrm{~mol})$ in methylene chloride $(200 \mathrm{~mL})$ under argon. $(1 S, 2 S)$-Pseudoephedrine $(15 \mathrm{~g}, 0.091 \mathrm{~mol})$ was then added portionwise over 15 min maintaining an internal temperature below $15^{\circ} \mathrm{C}$. After stirring with gradual warming to ambient temperature over 12 hours the methylene chloride was removed by distillation. The resulting residue was diluted with pentane $(150 \mathrm{~mL})$ and vigorously stirred for 4 hours to ensure complete precipitation of all triethylamine salts. Filtration of the suspension through a pad of celite and concentration of the filtrate by distillation afforded the crude product as a pale-orange oil. Purification by distillation under reduced pressure (bp $\sim 138{ }^{\circ} \mathrm{C}, 0.5$ mm Hg) gave $(S, S)-7$ ( 2:1 mixture of diastereomers) as a colorless oil (20 g, 75\%): ${ }^{1} \mathrm{H} \mathrm{NMR}(400 \mathrm{MHz}$, $\mathrm{CDCl}_{3}$ ) (major diastereomer) $\square 7.85(\mathrm{dd}, J=8.0,1.3 \mathrm{~Hz}, 2 \mathrm{H}, \mathrm{Ar}-\mathbf{H}), 7.30-7.56(\mathrm{~m}, 8 \mathrm{H}, \mathrm{Ar}-\mathbf{H}), 4.73(\mathrm{~d}, J=$ $8.3 \mathrm{~Hz}, 1 \mathrm{H}, \mathrm{PhCH}-\mathrm{O}), 3.17$ (dq, $\left.J=8.3,6.0 \mathrm{~Hz}, 1 \mathrm{H}, \mathrm{CH}_{3} \mathrm{CH}-\mathrm{N}\right), 2.53\left(\mathrm{~s}, 3 \mathrm{H}, \mathrm{CH}_{3}-\mathrm{N}\right), 1.20$ (d, $J=6.0 \mathrm{~Hz}$, $\left.3 \mathrm{H}, \mathrm{CH}_{3}-\mathrm{CHN}\right)$; (minor diastereomer) $\square 7.97$ (dd, $\left.J=8.0,1.4 \mathrm{~Hz}, 2 \mathrm{H}, \mathrm{Ar}-\mathbf{H}\right), 7.30-7.56$ (m, 8H, Ar-H), $4.86(\mathrm{~d}, J=7.2 \mathrm{~Hz}, 1 \mathrm{H}, \mathrm{PhCH}-\mathrm{O}), 3.34$ (q, $\left.J=6.2 \mathrm{~Hz}, 1 \mathrm{H}, \mathrm{CH}_{3} \mathrm{CH}-\mathrm{N}\right), 2.53\left(\mathrm{~s}, 3 \mathrm{H}, \mathrm{CH}_{3}-\mathrm{N}\right), 1.26(\mathrm{~d}, J=$ $\left.6.1 \mathrm{~Hz}, 3 \mathrm{H}, \mathrm{CH}_{3}-\mathrm{CHN}\right) ;{ }^{13} \mathrm{C}$ NMR $\left(100 \mathrm{MHz}, \mathrm{CDCl}_{3}\right) \square 141.0,140.6,134.8,134.7,131.55,131.48,130.1$, $128.2,128.0,127.94,127.87,127.8,126.6,126.2,125.8,85.8,84.2,63.9,62.8,29.7,29.4,17.4,16.8 ;{ }^{29} \mathrm{Si}$ NMR (60 MHz, $\left.\mathrm{CDCl}_{3}\right) \square-12.44$ (major diastereomer) and -13.85 (minor diastereomer); IR $\left(\mathrm{CHCl}_{3}\right)$ $3074,3033,2971,2890,2812,1592,1492,1448,1431,1373,1280,1215,1189,1122 \mathrm{~cm}^{-1}$.

Preparation of complex 11 (Figure 1): Hydrazone 8 (1.87 g, $8.36 \mathrm{mmol}$ ) was added to a solution of 7 (2.59 g, $8.52 \mathrm{mmol})$ in $\mathrm{CH}_{2} \mathrm{Cl}_{2}(10 \mathrm{~mL})$ under an atmosphere of argon. The resulting mixture was stirred at ambient temperature for $15 \mathrm{~min}$, and the $\mathrm{CH}_{2} \mathrm{Cl}_{2}$ was then removed by vacuum distillation. The ${ }^{1} \mathrm{H}$ NMR spectrum of this material is a mixture of 4 compounds, all displaying characteristics of complexes 9 and/or 10 (tentative structural assignments; the spectrum is reproduced below). The residue was diluted with toluene $(10 \mathrm{~mL})$ and the mixture stirred for $12 \mathrm{~h}$ under argon, during which time the precipitation of a white solid was observed. The suspension was transferred to an inert atmosphere glove box and filtered, washed ( $3 \times 5 \mathrm{~mL}$ toluene), and dried under vacuum to yield the desired compound $\mathbf{1 1}$ (as a single compound) as a white solid (4.0 g, 90\% yield). Crystals suitable for X-ray analysis were grown over 2 days by layering hexanes $(3.0 \mathrm{~mL})$ over a solution of compound $11(175 \mathrm{mg}, 0.33 \mathrm{mmol})$ in $\mathrm{CH}_{2} \mathrm{Cl}_{2}(1.0$ 
mL). Data for 11: ${ }^{1} \mathrm{H}$ NMR (400 MHz, $\left.\mathrm{CDCl}_{3}\right) \square 9.92(\mathrm{~m}, 1 \mathrm{H}, \mathrm{MeN}-\mathbf{H}), 8.52$ (d, J = 6.3 Hz, 2H, Ar-H), $8.28(\mathrm{~d}, J=7.3 \mathrm{~Hz}, 2 \mathrm{H}, \mathrm{Ar}-\mathbf{H}), 7.89(\mathrm{~s}, 1 \mathrm{H}, \mathrm{N}=\mathrm{C}-\mathbf{H}), 7.58-7.68(\mathrm{~m}, 8 \mathrm{H}, \mathrm{Ar}-\mathbf{H}), 7.45-7.55$ (m, 8H, Ar-H), $5.47(\mathrm{~d}, J=10.2 \mathrm{~Hz}, 1 \mathrm{H}, \mathrm{PhCH}-\mathrm{O}), 3.04-3.15\left(\mathrm{~m}, 1 \mathrm{H}, \mathrm{CH}_{3} \mathrm{CH}-\mathrm{N}\right), 2.40$ (d, J = 5.4 Hz, 3H, CH $\left.-\mathrm{N}\right), 1.50$ $\left(\mathrm{d}, J=6.4 \mathrm{~Hz}, 3 \mathrm{H}, \mathrm{CH}_{3}-\mathrm{CHN}\right) ;{ }^{13} \mathrm{C} \mathrm{NMR}\left(100 \mathrm{MHz}, \mathrm{CDCl}_{3}\right) \square 167.5,156.2,137.8,134.24,134.18$, $133.3,132.5,131.9,131.3,130.6,129.1,128.9,128.7,128.66,128.60 .128 .2,127.0,126.7,126.3,79.3$, 61.9, 32.9, 11.2; ${ }^{29} \mathrm{Si} \mathrm{NMR}\left(60 \mathrm{MHz}, \mathrm{CDCl}_{3}\right) \square-89.10$.

Determination of enantioselectivity of homoallylic hydrazide products: All ee's were determined by chiral HPLC analysis. Racemic samples were prepared by the method of Kobayashi. ${ }^{4}$

Table 1, entry 1: Chiralpak AD-H Column, 20.0\% EtOH/hexanes, $1.0 \mathrm{ml} / \mathrm{min}, 220 \mathrm{~nm}$.

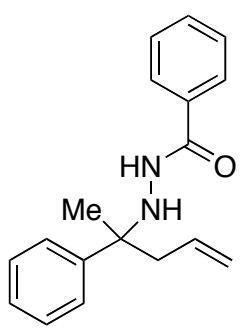

Racemic

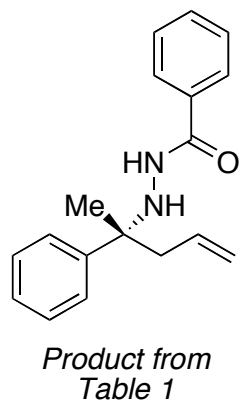

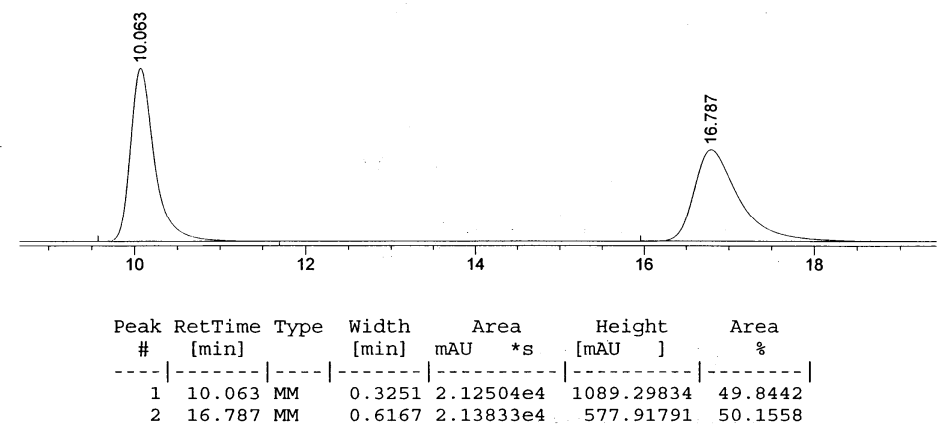

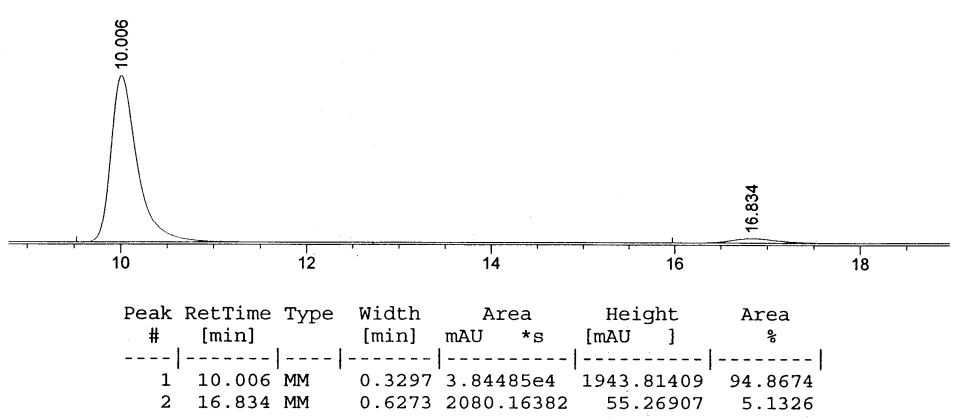

(4) Hirabayashi, R.; Ogawa, C.; Sugiura, M.; Kobayashi, S. J. Am. Chem. Soc. 2001, 123, 9493-9499. 
Table 1, entry 2: Chiralpak AD-H Column, 25.0\% EtOH/hexanes, $1.0 \mathrm{ml} / \mathrm{min}, 220 \mathrm{~nm}$.<smiles>C=CCC(CC)(NNC(=O)c1ccccc1)c1ccccc1</smiles>

Racemic<smiles>C=CC[C@@](CC)(NNC(=O)c1ccccc1)c1ccccc1</smiles>

Product from Table 1
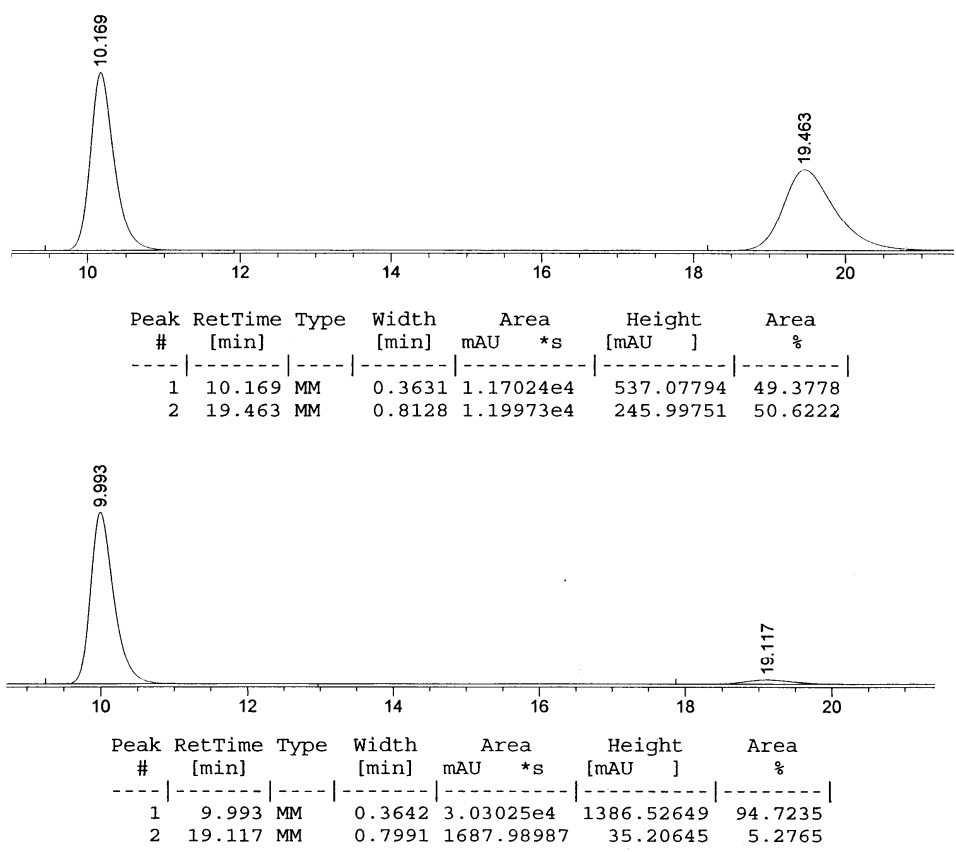

Table 1, entry 3: Chiralpak AD-H Column, 15.0\% EtOH/hexanes, $1.0 \mathrm{ml} / \mathrm{min}, 220 \mathrm{~nm}$.<smiles>C=CCC(Br)(NNC(=O)c1ccccc1)c1ccccc1</smiles>

Racemic<smiles>C=CC[C@](Br)(NNC(=O)c1ccccc1)c1ccccc1</smiles>

Product from Table 1
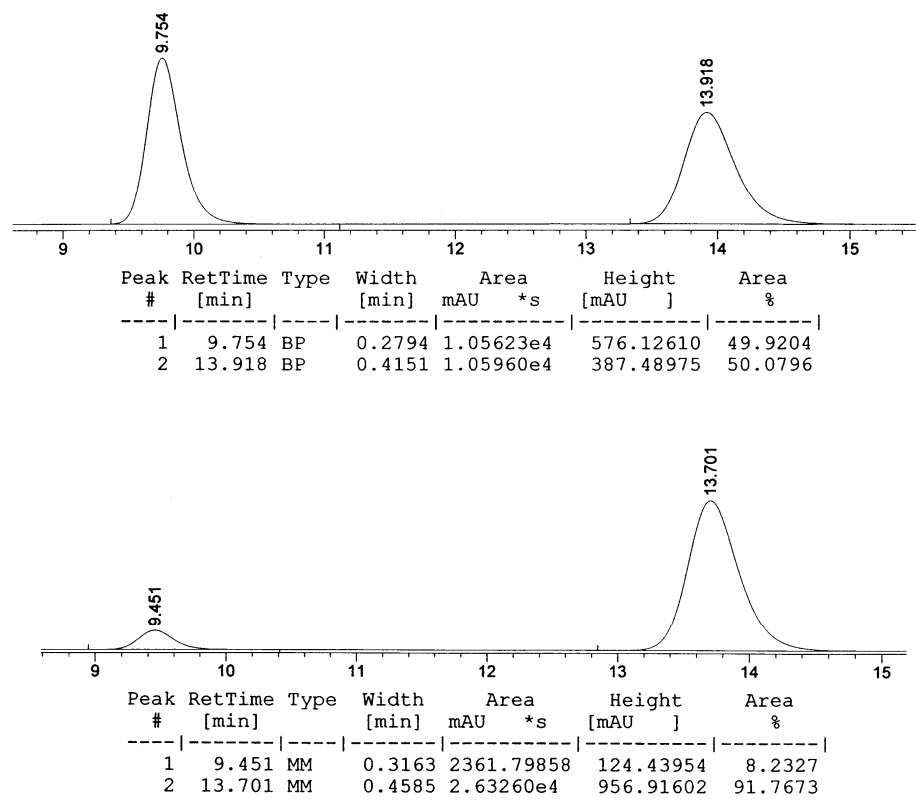
Table 1, entry 4: Chiralpak AD-H Column, 5.0\% EtOH/hexanes, $1.0 \mathrm{ml} / \mathrm{min}, 220 \mathrm{~nm}$.<smiles>C=CCC(NNC(=O)c1ccccc1)(c1ccccc1)C(F)(F)F</smiles><smiles>C=CC[C@](NNC(=O)c1ccccc1)(c1ccccc1)C(F)(F)c1ccccc1</smiles>
Table 1
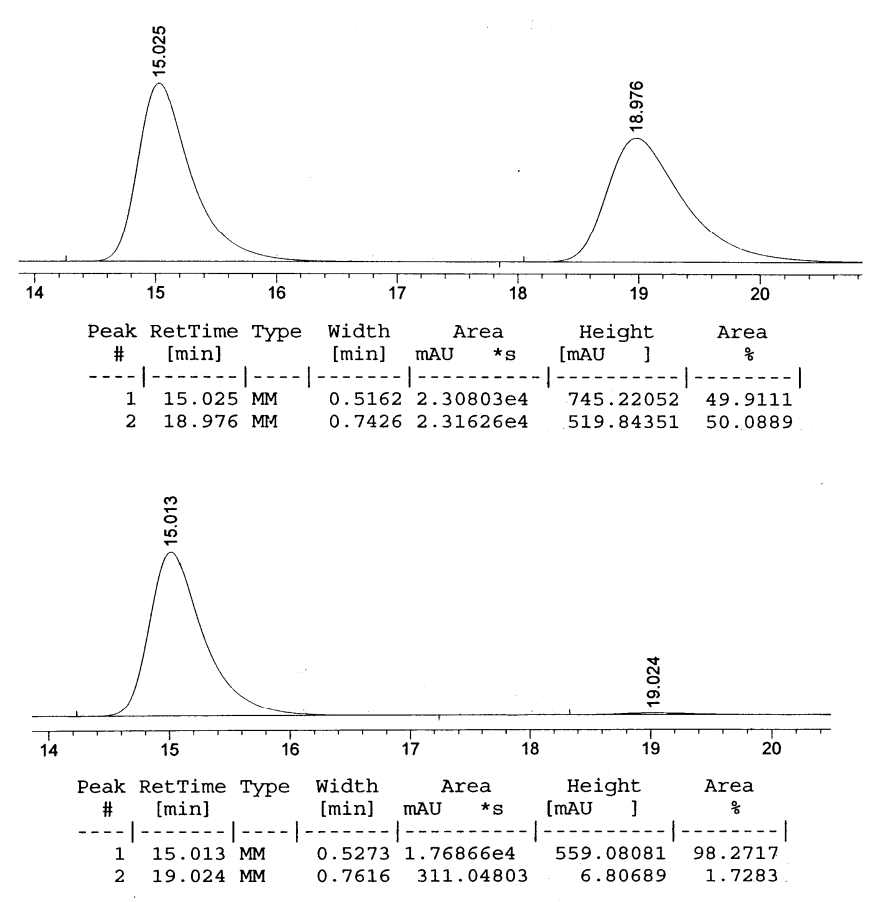

Table 1, entry 5: Chiralpak AD-H Column, 10.0\% IPA/hexanes, $1.0 \mathrm{ml} / \mathrm{min}, 220 \mathrm{~nm}$.<smiles>C=CCC(CC=C)(NNC(=O)c1ccccc1)c1ccccc1</smiles>

Racemic<smiles>C=CCC(NC(=O)c1ccccc1)(c1ccccc1)c1ccccc1</smiles>
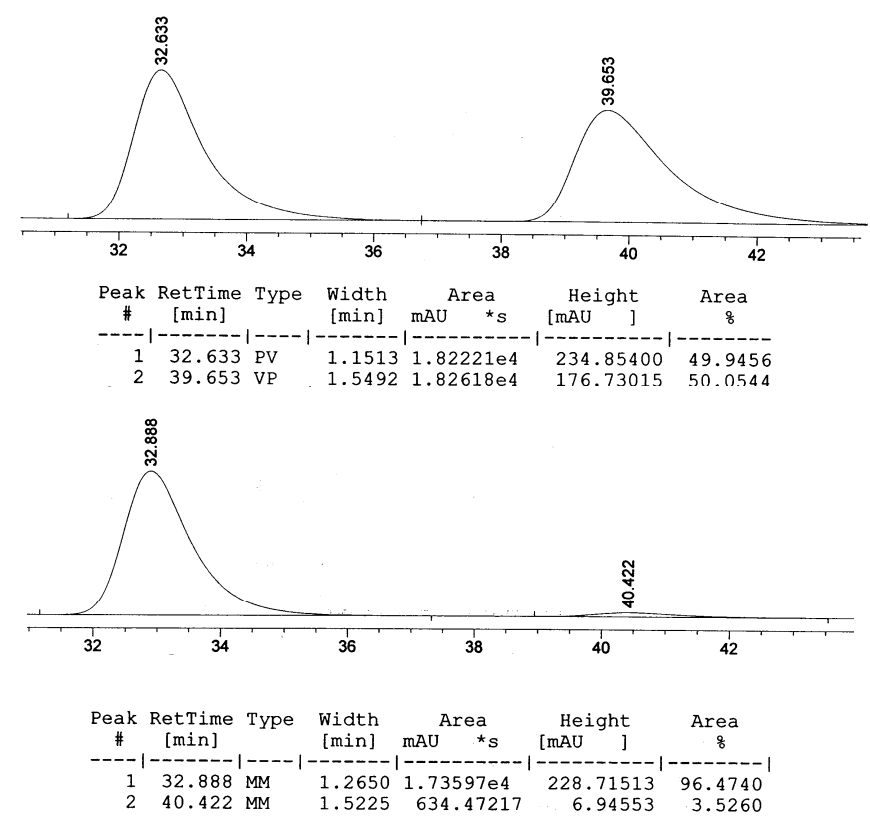
Table 1, entry 6: Chiralpak AD-H Column, 15.0\% IPA/hexanes, 1.0 ml/min, $220 \mathrm{~nm}$.<smiles>C=CCC(C)(NNC(=O)c1ccccc1)c1ccc(Br)cc1</smiles>

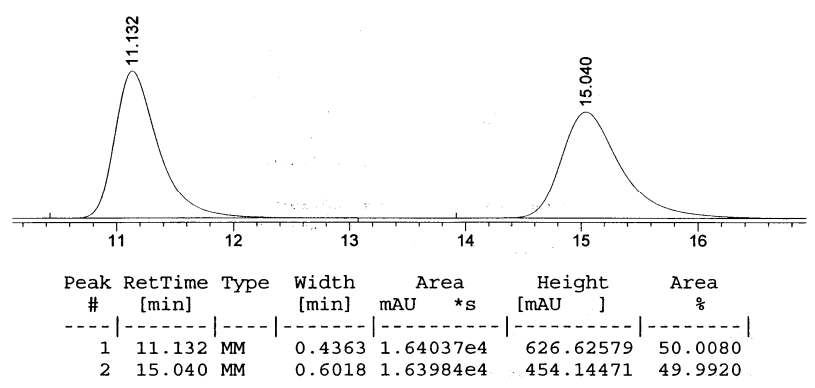<smiles>C=CC[C@](C)(NNC(=O)c1ccccc1)c1ccc(Br)cc1</smiles>

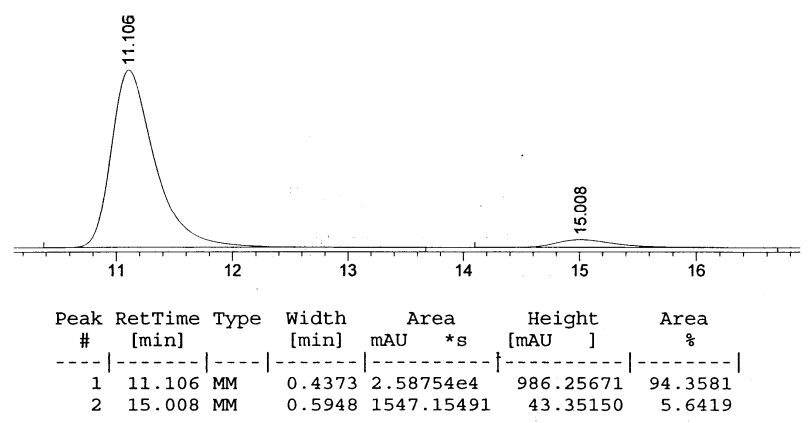

Table 1, entry 7: Chiralpak AD-H Column, 20.0\% EtOH/hexanes, $1.0 \mathrm{ml} / \mathrm{min}, 220 \mathrm{~nm}$.<smiles>C=CCC(NNC(=O)c1ccccc1)(c1ccc(OC)cc1)[N+](C)(C)[O-]</smiles>

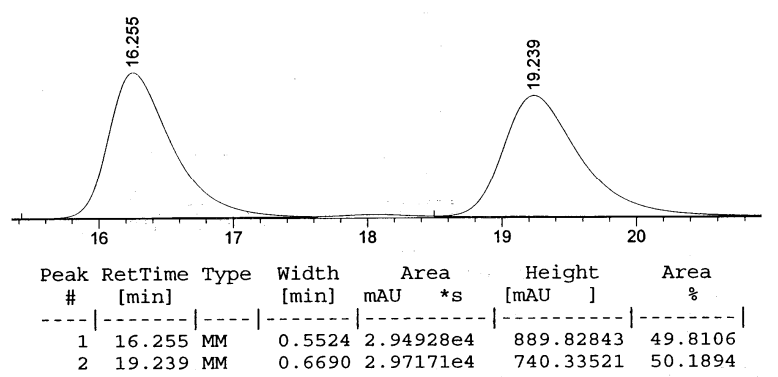<smiles>C=CC[C@@](C)(NNC(=O)c1ccccc1)c1ccc(OC)cc1</smiles>

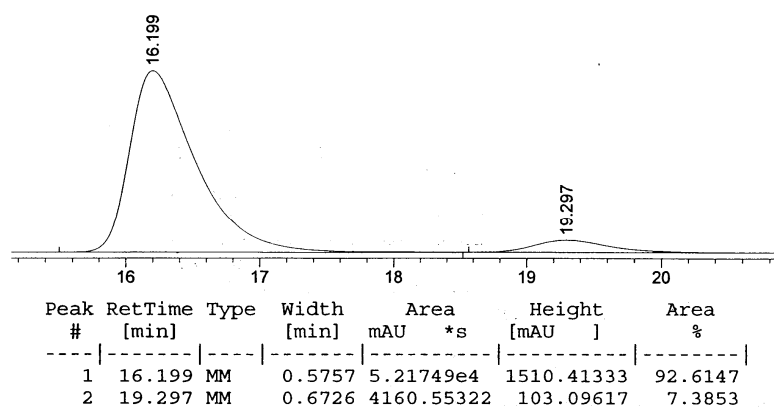


Table 1, entry 8: Chiralpak AD-H Column, $20.0 \%$ EtOH/hexanes, $1.0 \mathrm{ml} / \mathrm{min}, 220 \mathrm{~nm}$.<smiles>C=CCC(C)(NNC(=O)c1ccccc1)c1cccc([N+](=O)[O-])c1</smiles>

Racemic<smiles>C=CC[C@](C)(NNC(=O)c1ccccc1)c1cccc([N+](=O)[O-])c1</smiles>

Product from Table 1

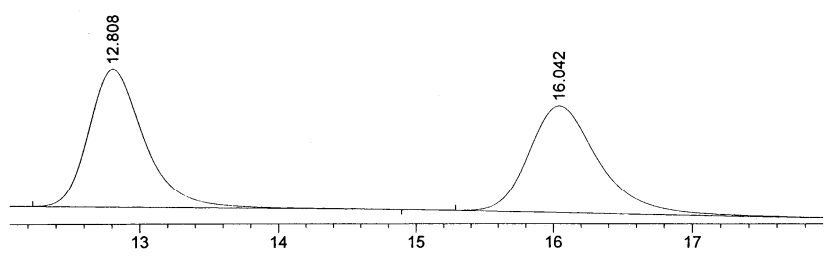

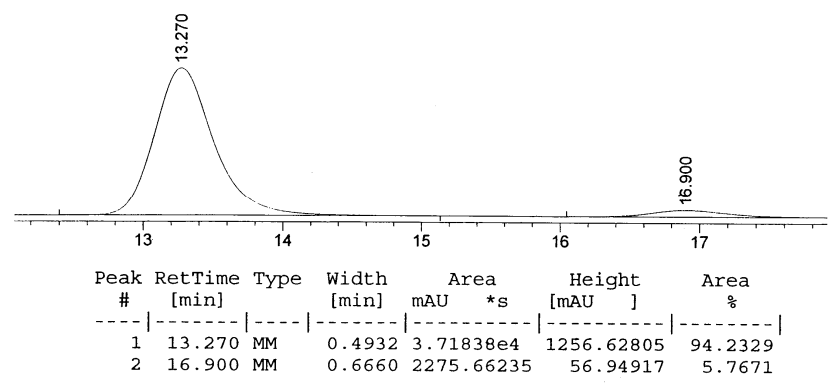

Table 1, entry 9: Chiralpak AD-H Column, 20.0\% EtOH/hexanes, $1.0 \mathrm{ml} / \mathrm{min}, 220 \mathrm{~nm}$.<smiles>C=CCC(C)(NNC(=O)c1ccccc1)c1ccc2ccccc2c1</smiles>

Racemic<smiles>C=CC[C@](C)(NNC(=O)c1ccccc1)c1ccc2ccccc2c1</smiles>
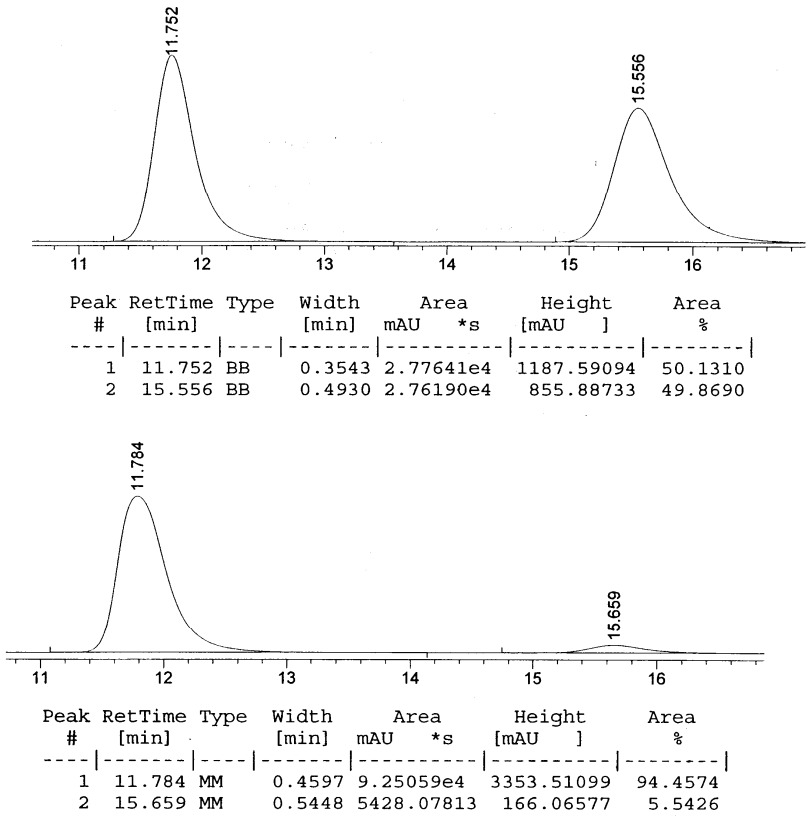
Table 1, entry 10: Chiralpak AD-H Column, 20.0\% EtOH/hexanes, $1.0 \mathrm{ml} / \mathrm{min}, 220 \mathrm{~nm}$.<smiles>C=CCC(C)(NNC(=O)c1ccccc1)c1ccco1</smiles>

Racemic<smiles>C=CC[C@](C)(NNC(=O)c1ccccc1)c1ccco1</smiles>

Product from

Table 1
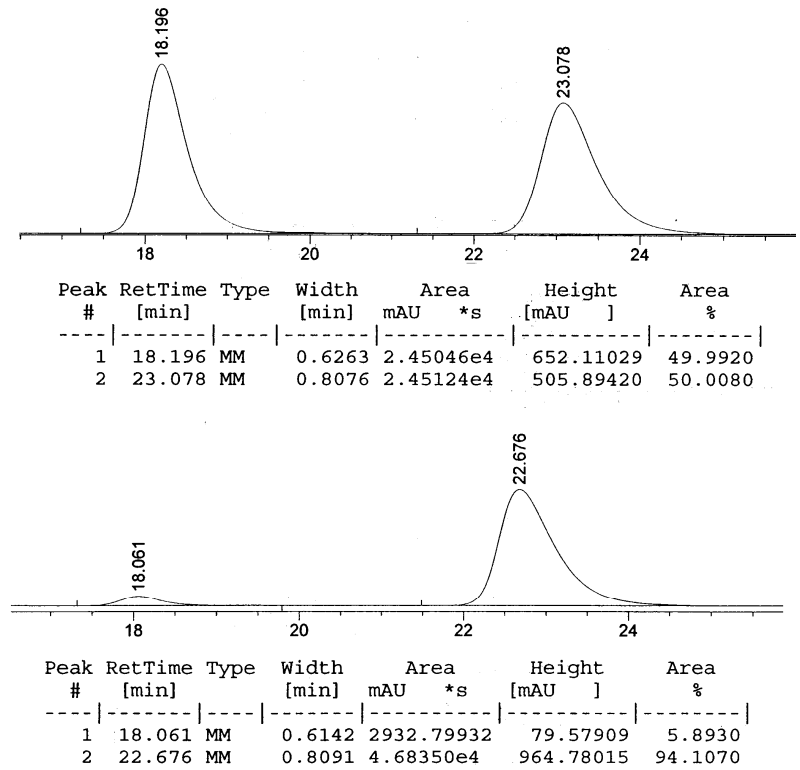

Table 1, entry 11: Chiralpak AD-H Column, 15.0\% IPA/hexanes, $1.0 \mathrm{ml} / \mathrm{min}, 220 \mathrm{~nm}$.

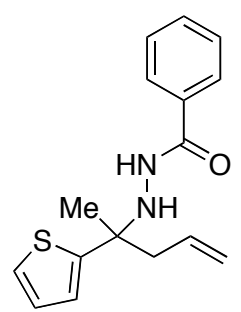

Racemic<smiles>C=CC[C@](C)(NNC(=O)c1ccccc1)c1cccs1</smiles>

Product from Table 1
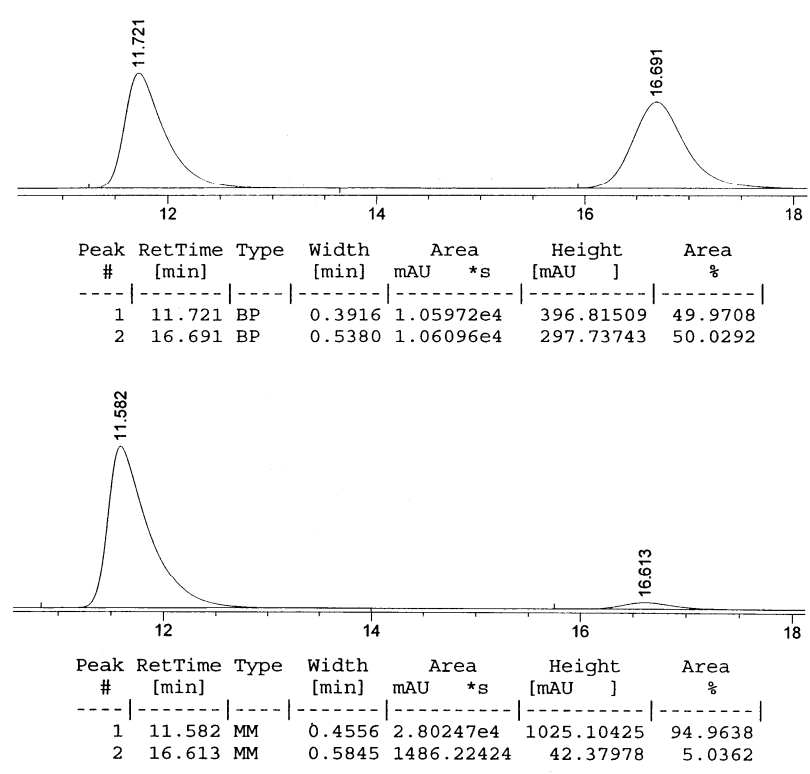
Table 1, entry 12: Chiralpak AD-H Column, 25.0\% EtOH/hexanes, $0.4 \mathrm{ml} / \mathrm{min}, 220 \mathrm{~nm}$.<smiles>C=CCC(C)(NNC(=O)c1ccccc1)c1cn(C(=O)OCc2ccccc2)c2ccccc12</smiles>
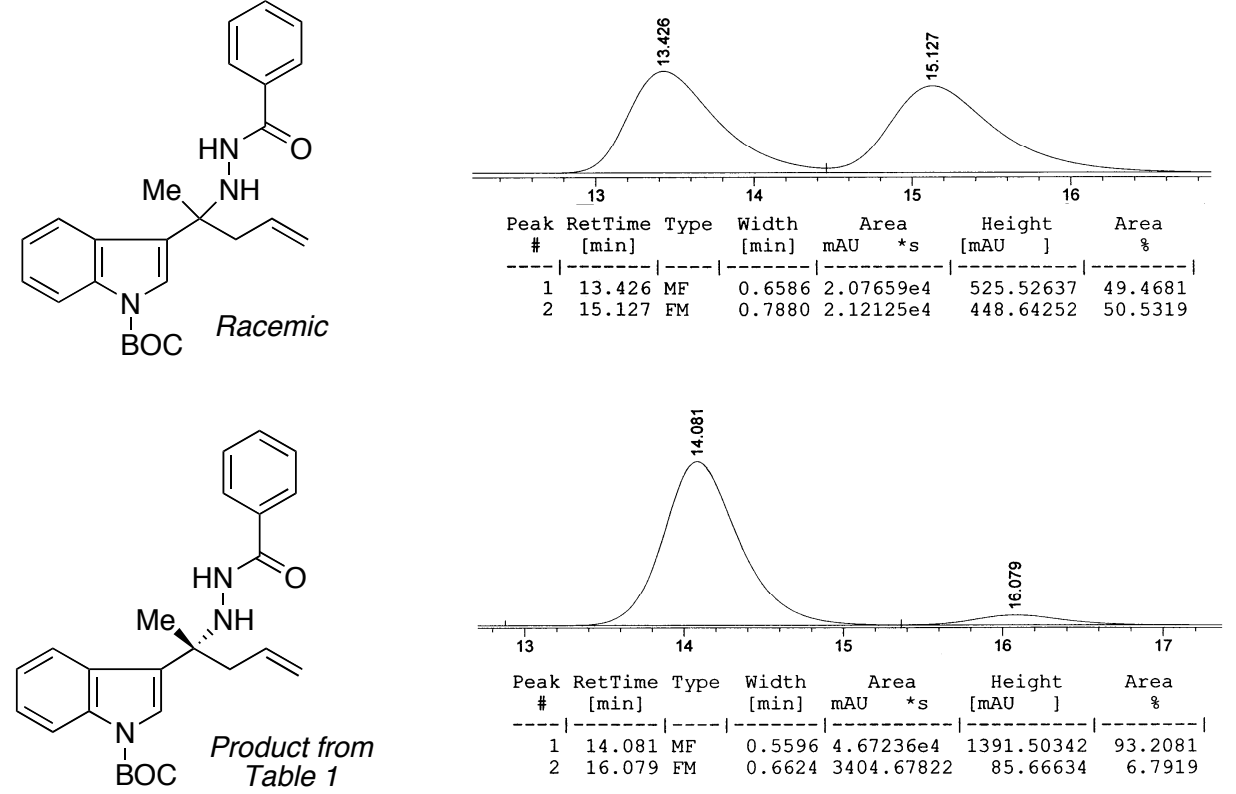

Table 1, entry 13: Chiralpak AD-H Column, 3.0\% MeOH:EtOH (1:1)/hexanes, $1.0 \mathrm{ml} / \mathrm{min}, 220 \mathrm{~nm}$,<smiles>C=CCC(C)(CCc1ccccc1)NNC(=O)c1ccccc1</smiles>
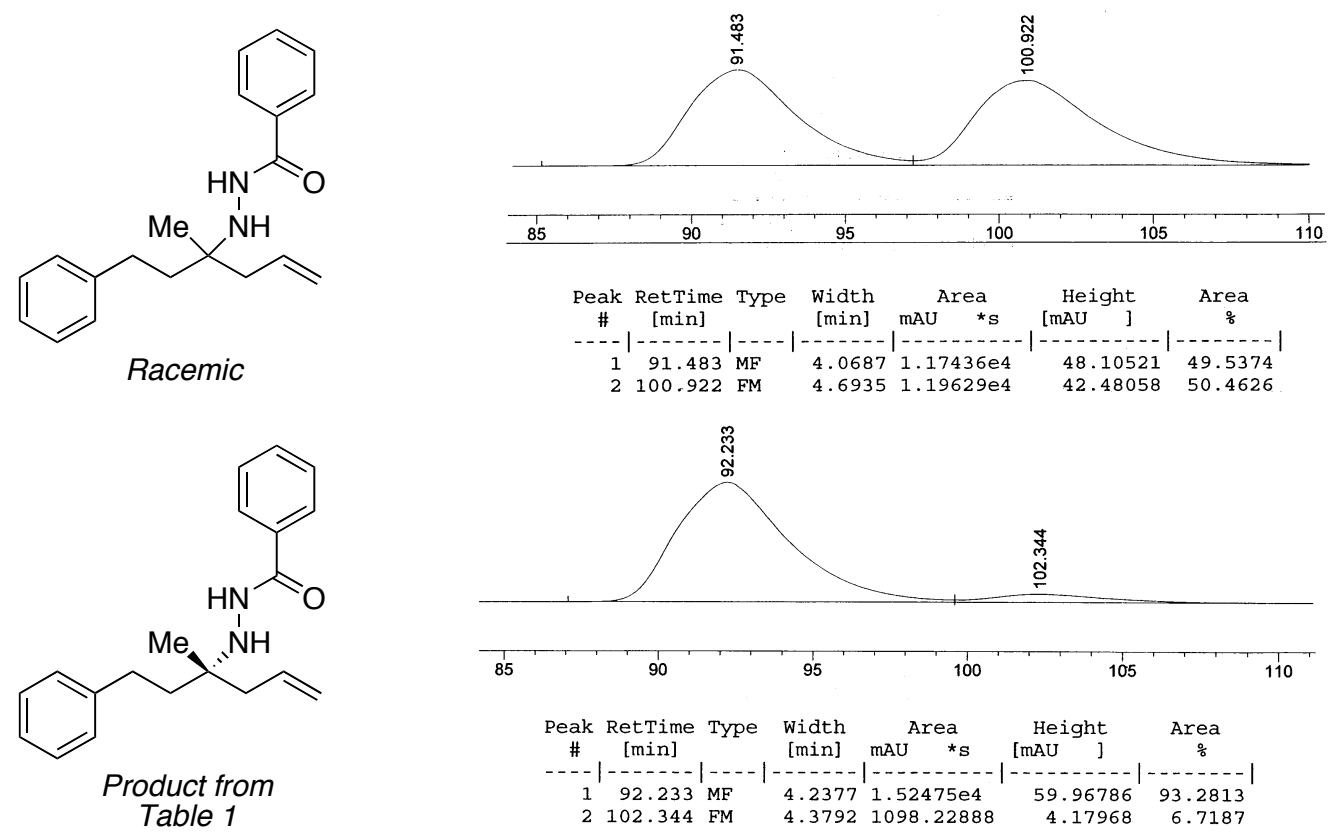
Table 1, entry 14: Chiralpak AD-H Column, 7.0\% IPA/hexanes, $1.0 \mathrm{ml} / \mathrm{min}, 220 \mathrm{~nm}$<smiles>C=CCC(C)(NNC(=O)c1ccccc1)C1CCCCC1</smiles>

Racemic<smiles>C=CC[C@@](C)(NNC(=O)c1ccccc1)C1CCCCC1</smiles>

Product from Table 1
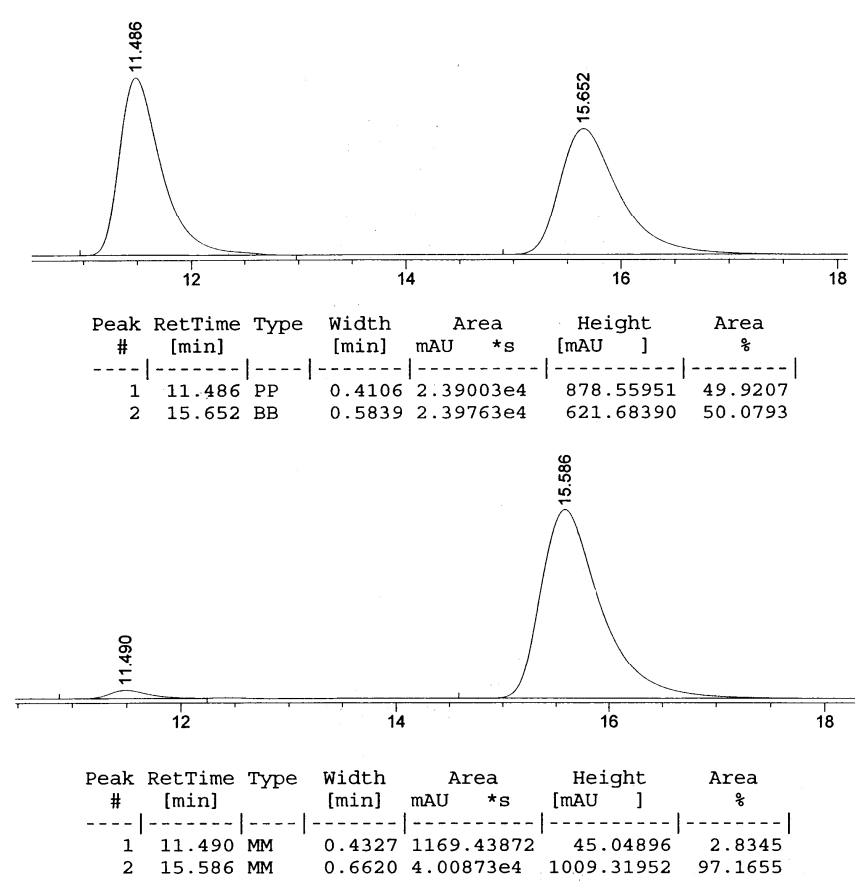

Scheme 2 (reaction in $\mathrm{CHCl}_{3}$ ): Chiralpak AD-H Column, 25.0\% EtOH/hexanes, $1.0 \mathrm{ml} / \mathrm{min}, 220 \mathrm{~nm}$.<smiles>C=CCC(C)(NNC(=O)c1ccccc1)c1ccccc1</smiles>

Racemic<smiles>C=CC[C@@](C)(NNC(=O)c1ccccc1)c1ccccc1</smiles>

Product from Scheme 2
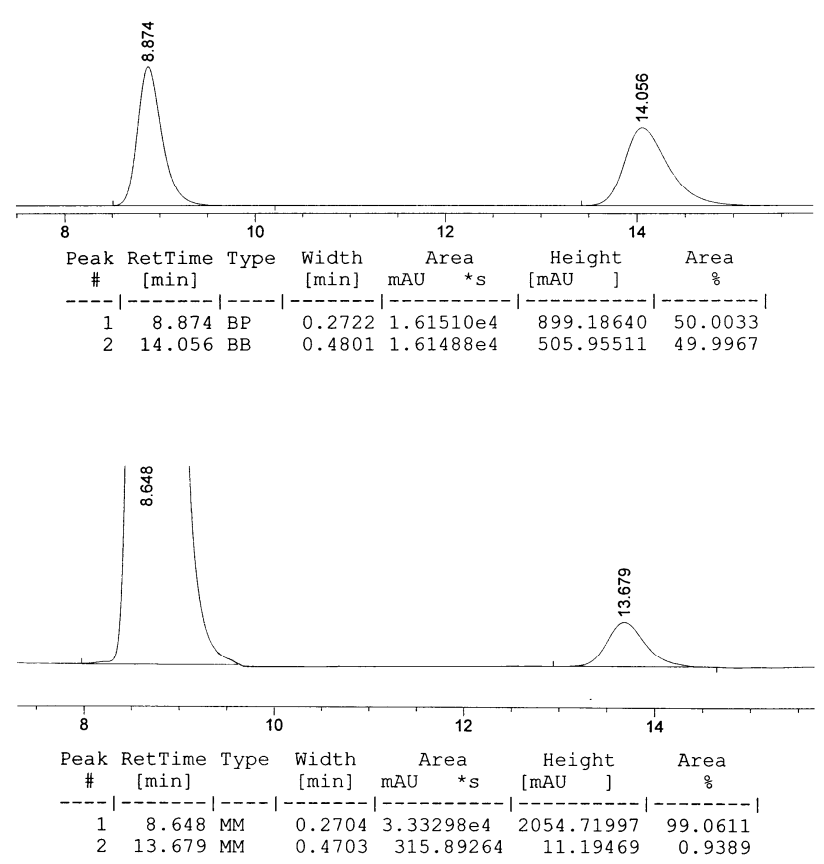
Page S20

${ }^{1} \mathrm{H}$ NMR (400 MHz, $\mathrm{CDCl}_{3}$ ) spectra of the allylation products from Table 1:
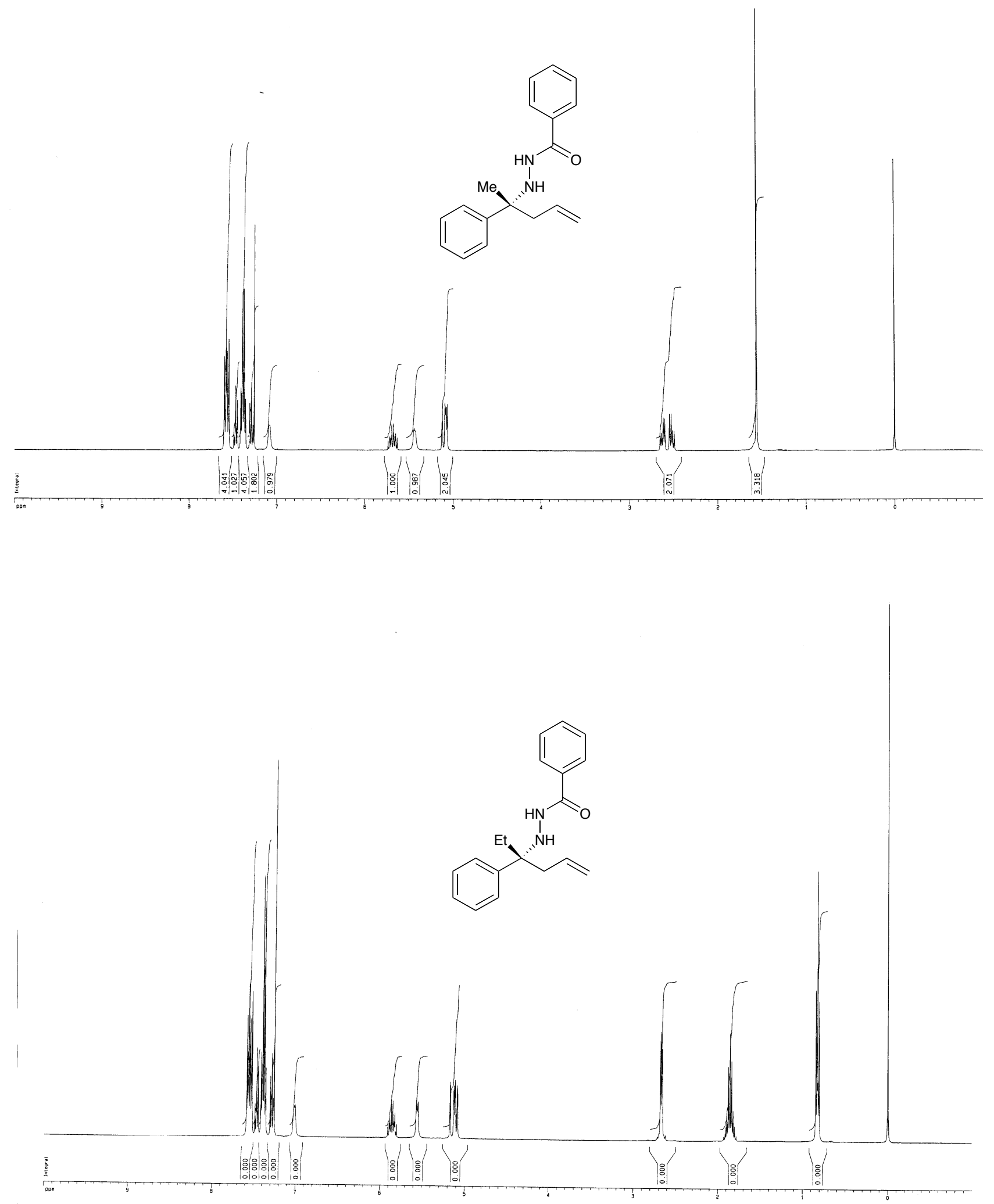
Page S21
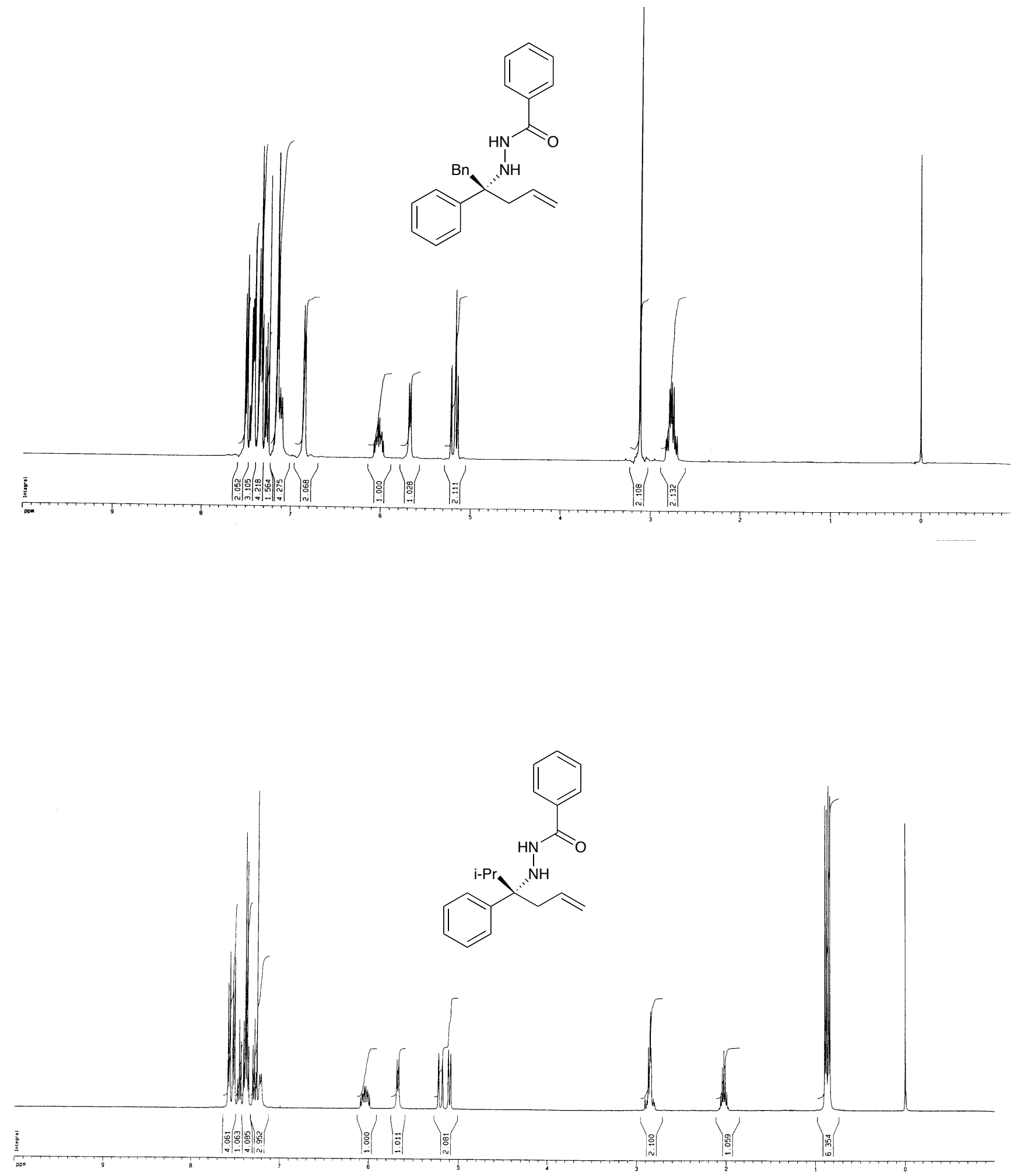
Page S22
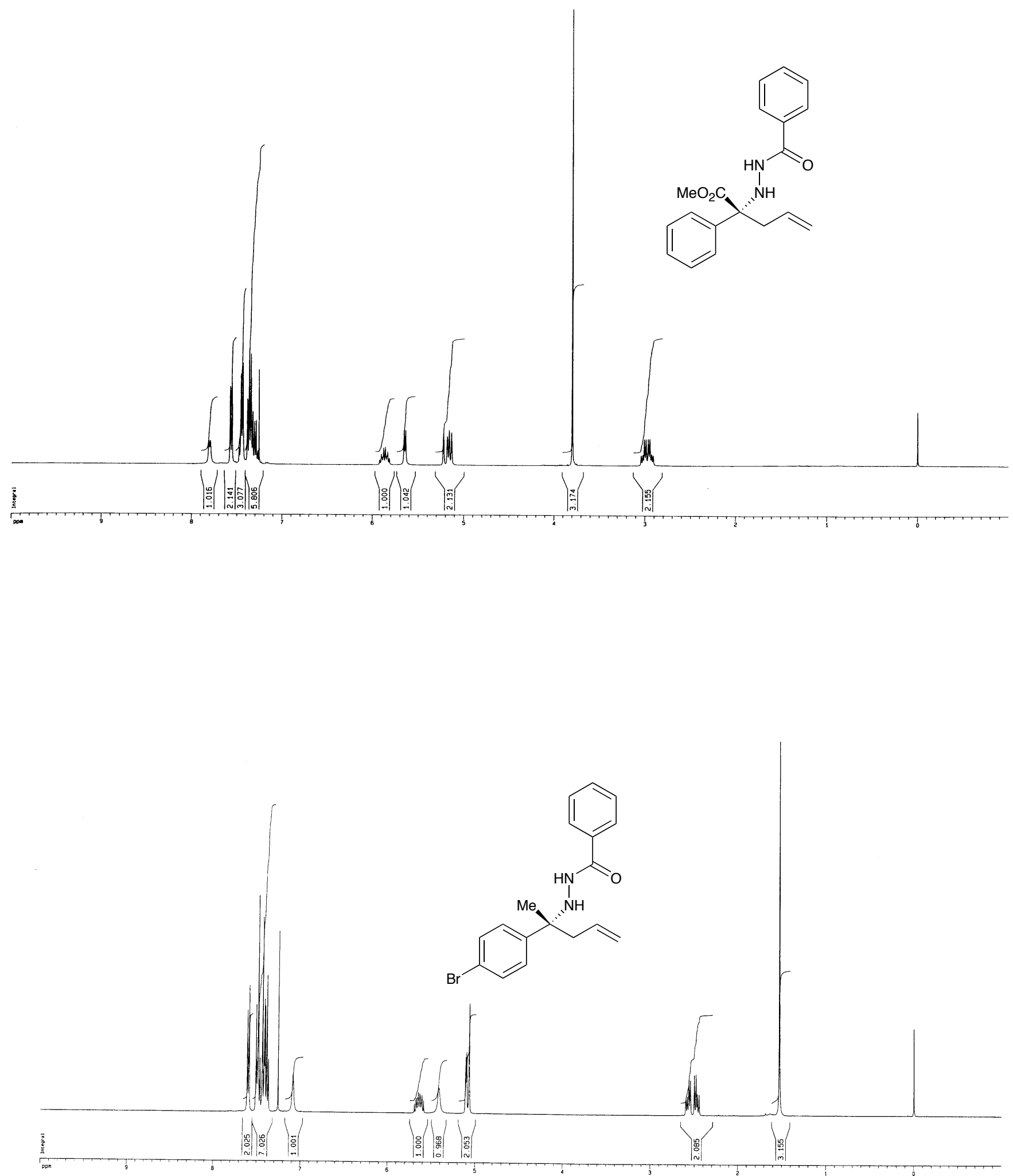
Page S23
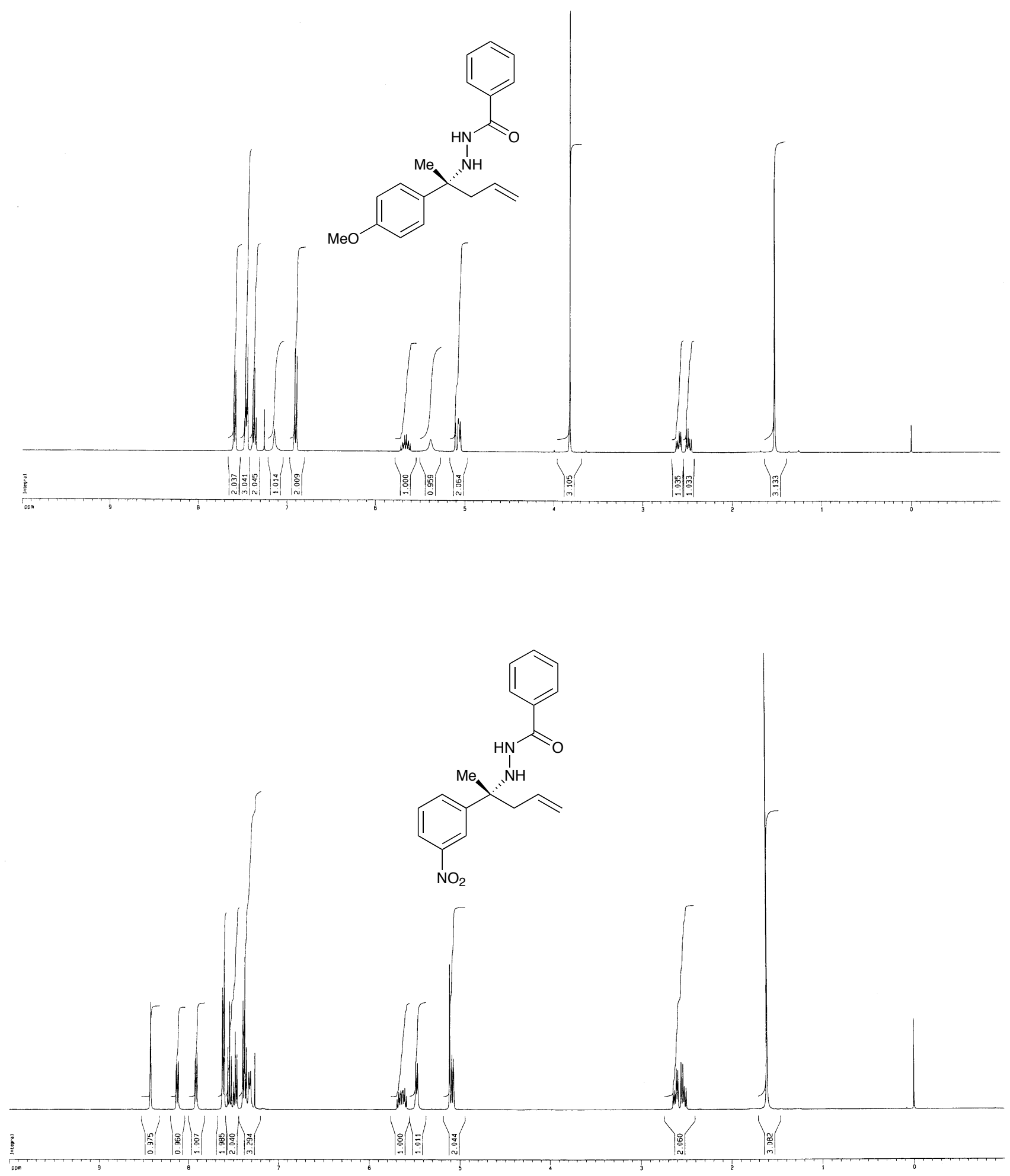
Page S24
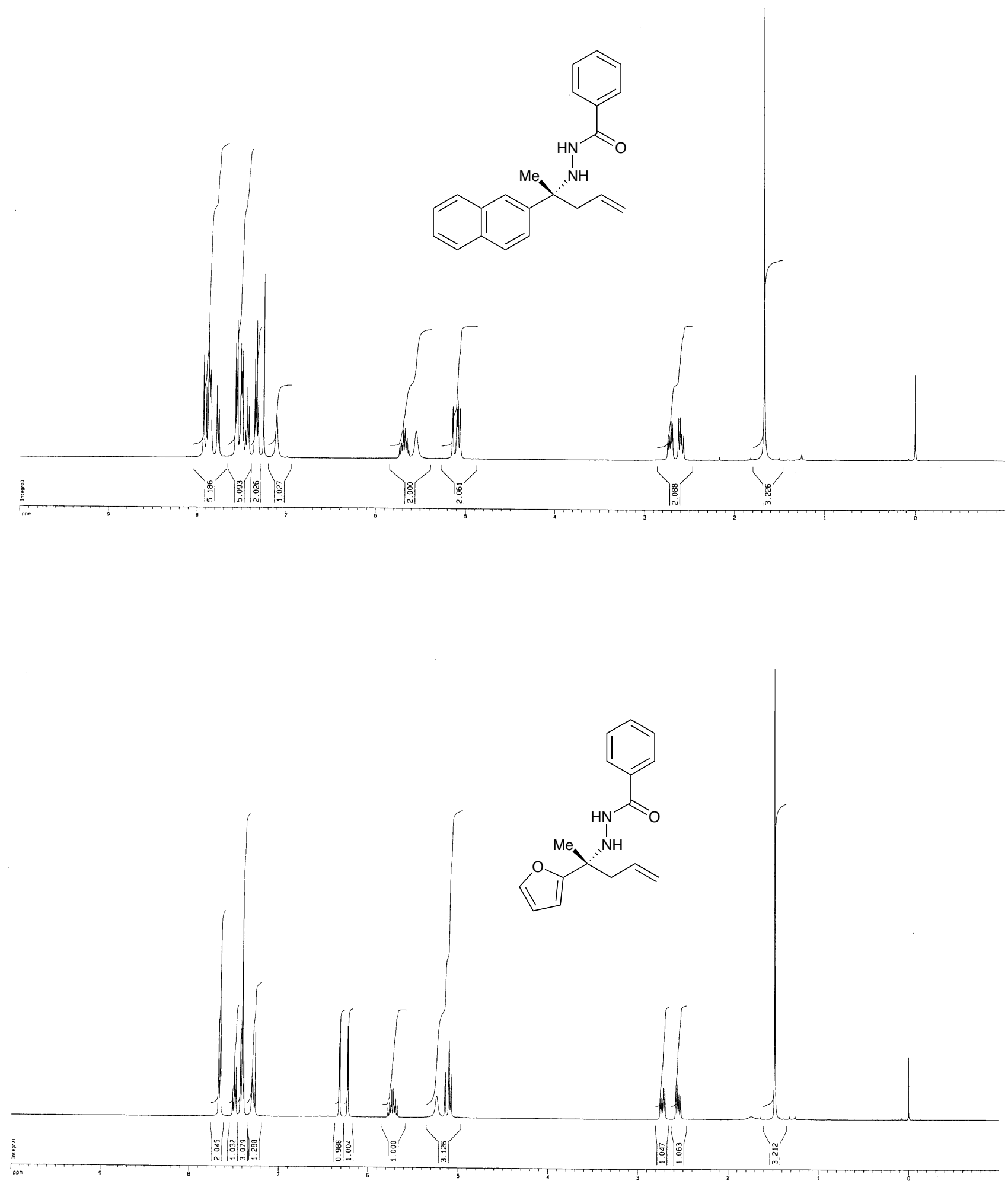
Page S25
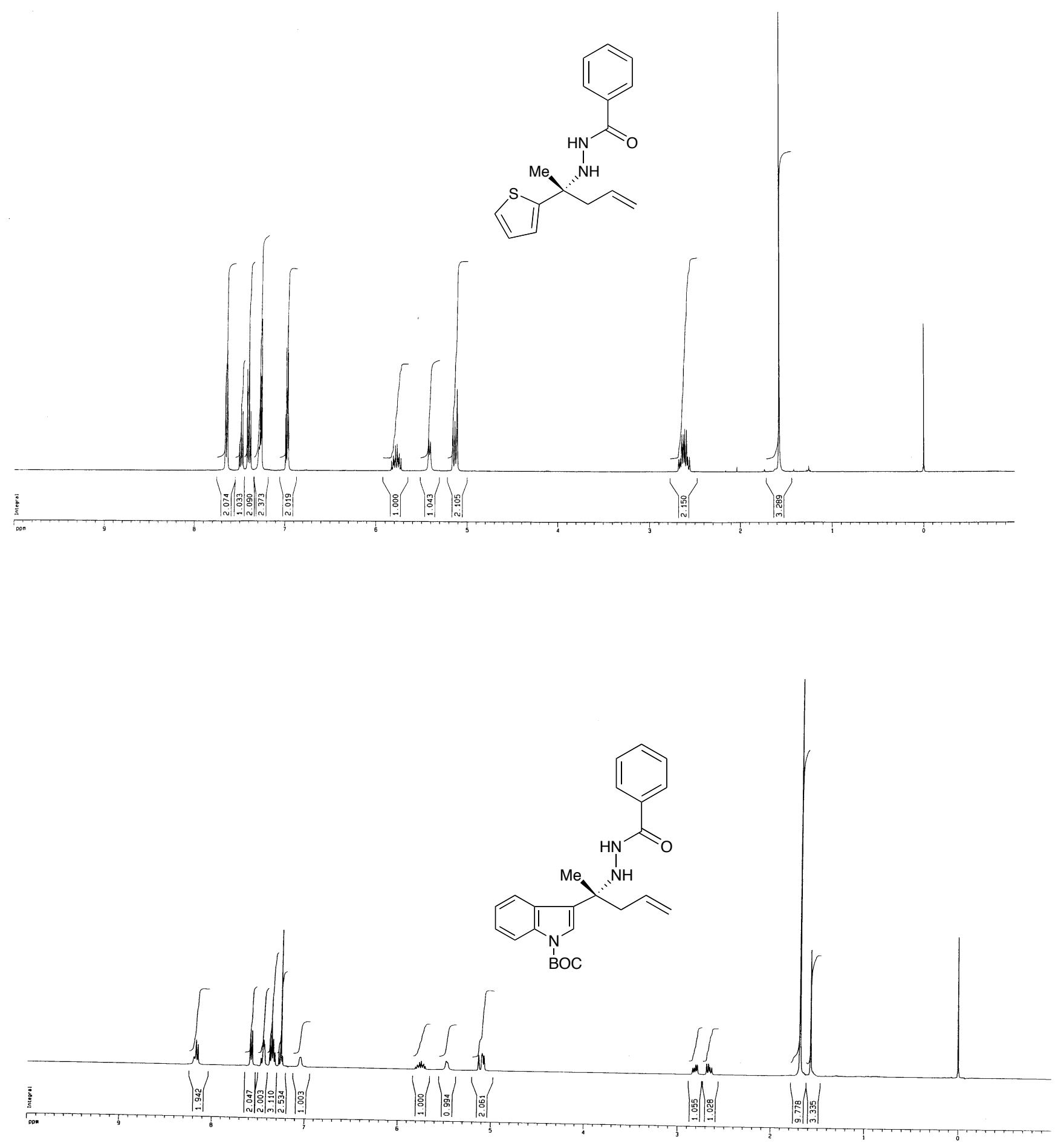
Page S26
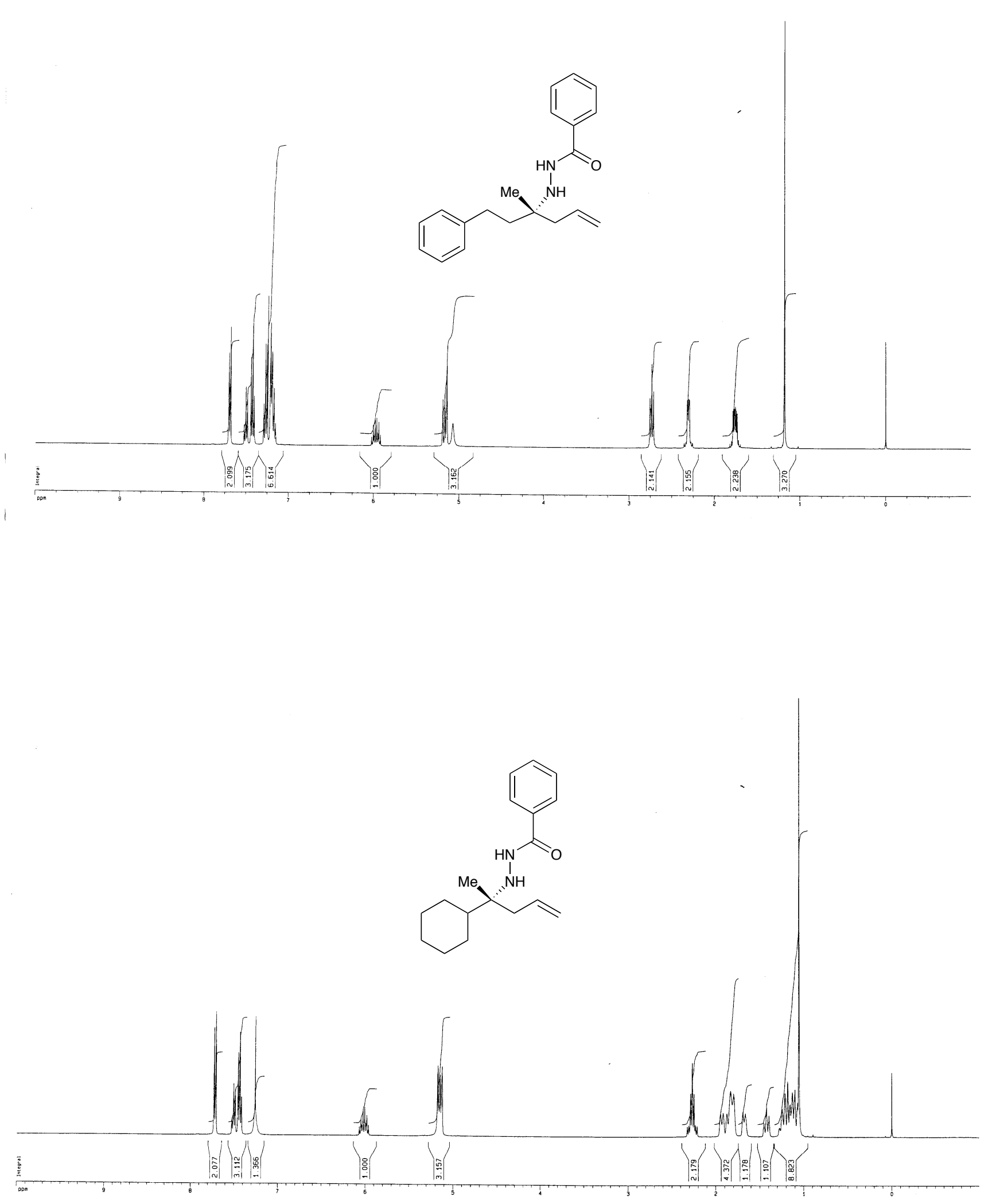
${ }^{1} \mathrm{H}$ NMR (400 MHz, DMSO- $d_{6}$ ) spectrum of the scale-up allylation product:

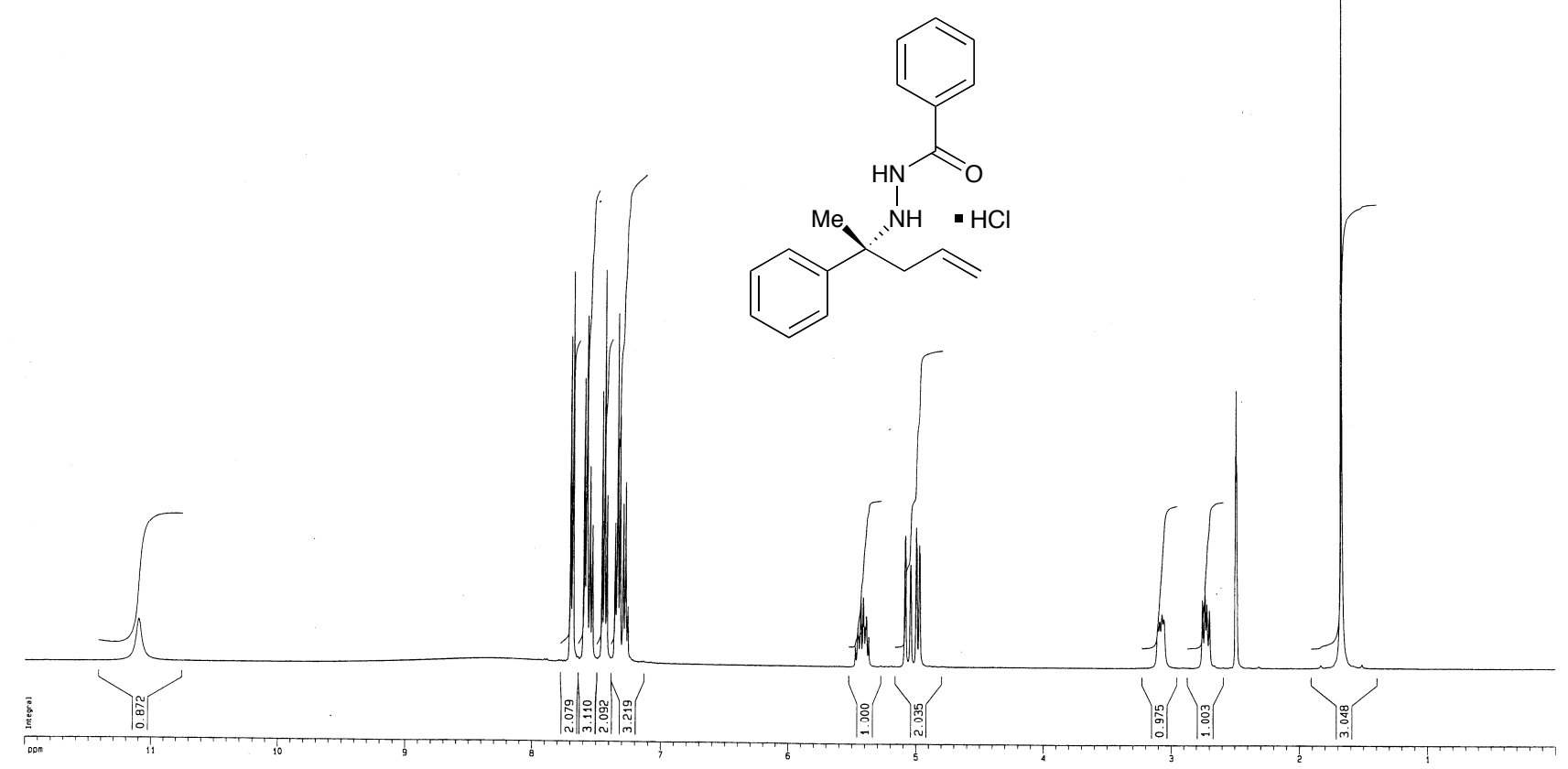

${ }^{1} \mathrm{H}$ NMR (400 MHz, $\mathrm{CDCl}_{3}$ ) spectrum of silane 7:

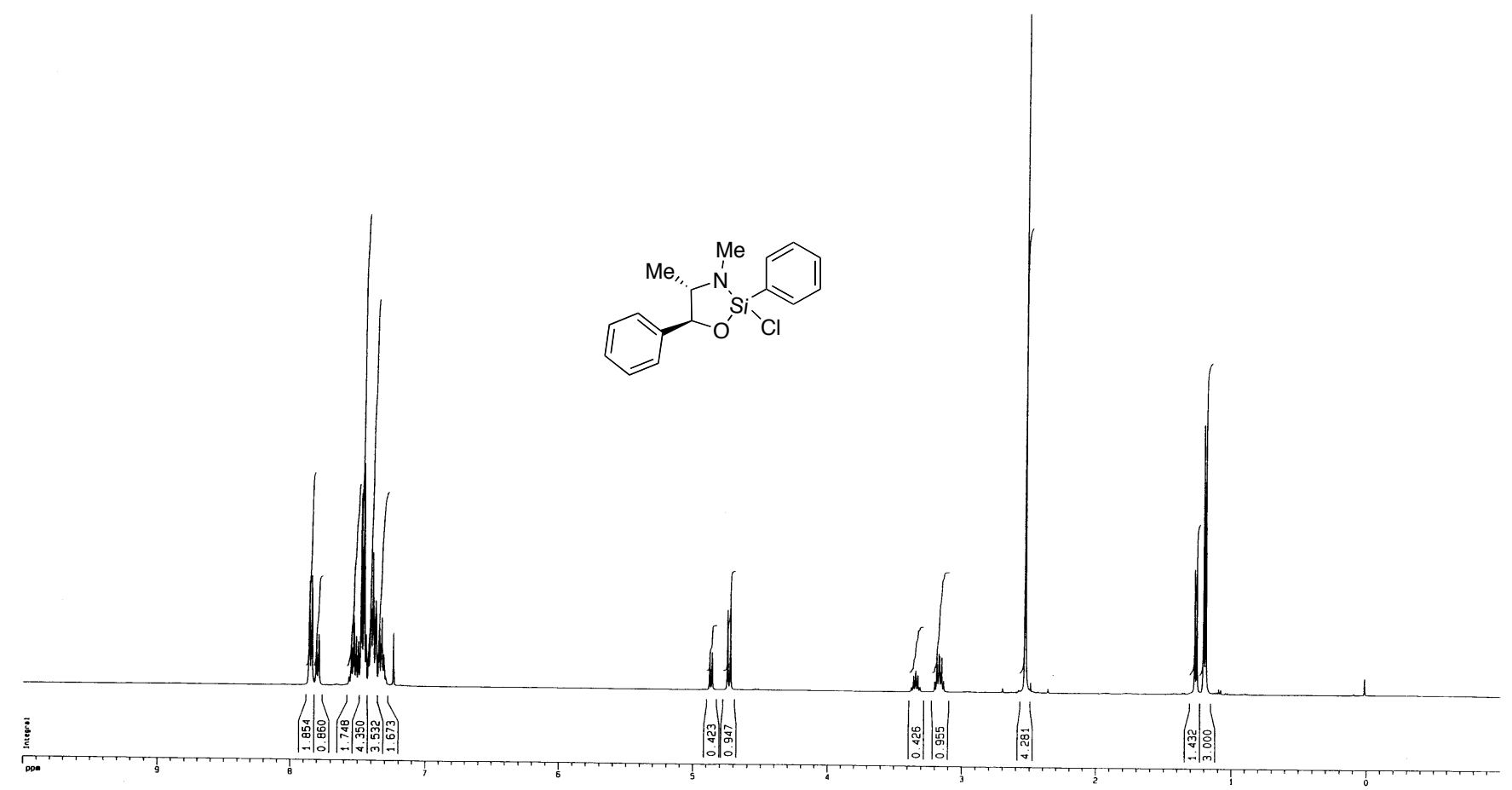


${ }^{29} \mathrm{Si} \mathrm{NMR}\left(60 \mathrm{MHz}, \mathrm{CDCl}_{3}\right)$ spectrum of silane 7:

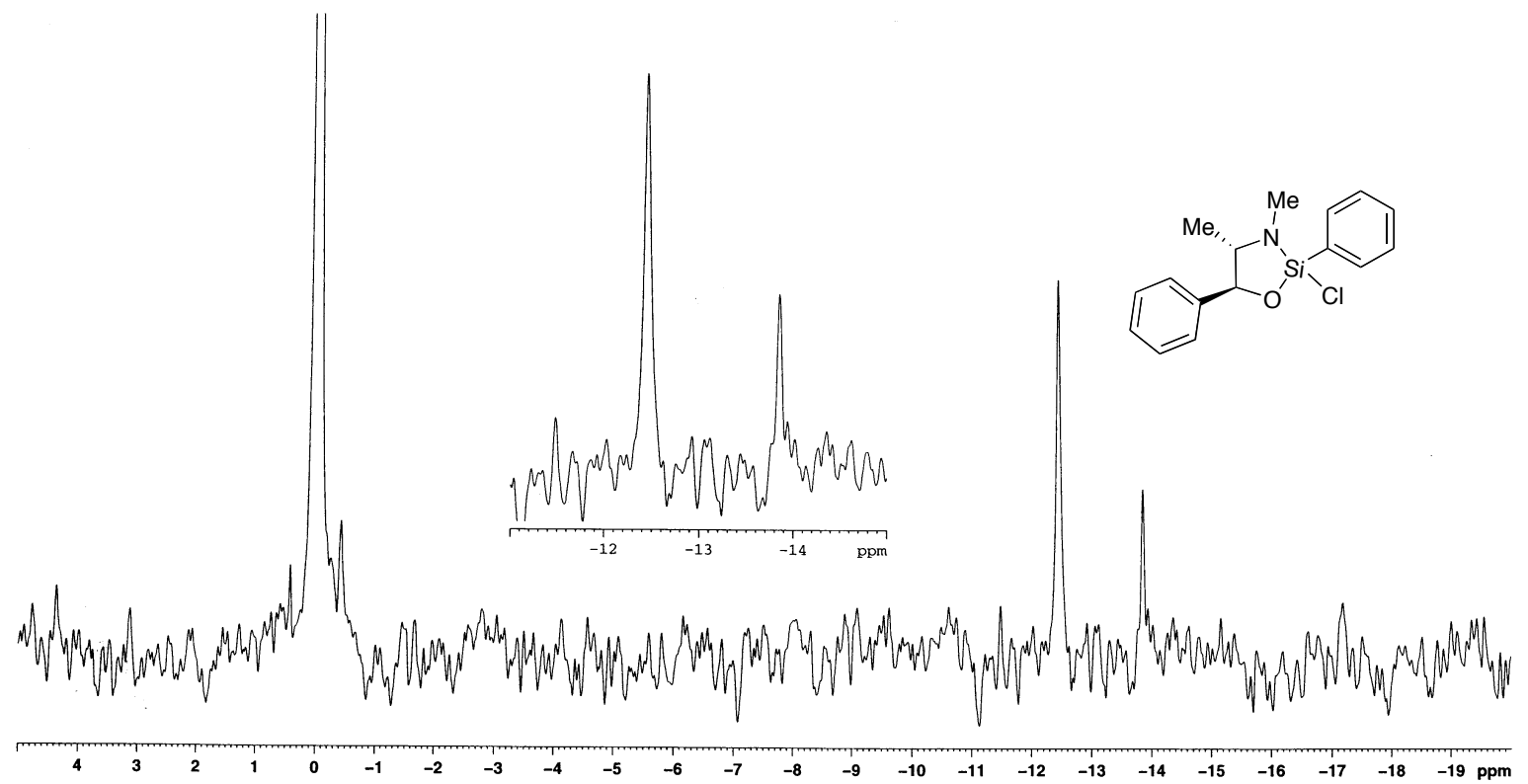

${ }^{1} \mathrm{H}$ NMR (400 MHz, $\mathrm{CDCl}_{3}$ ) spectrum of 9 and/or $\mathbf{1 0}$ (tentative structural assignment; the spectrum is consistent with the amine of the pseudoephedrine being protonated):

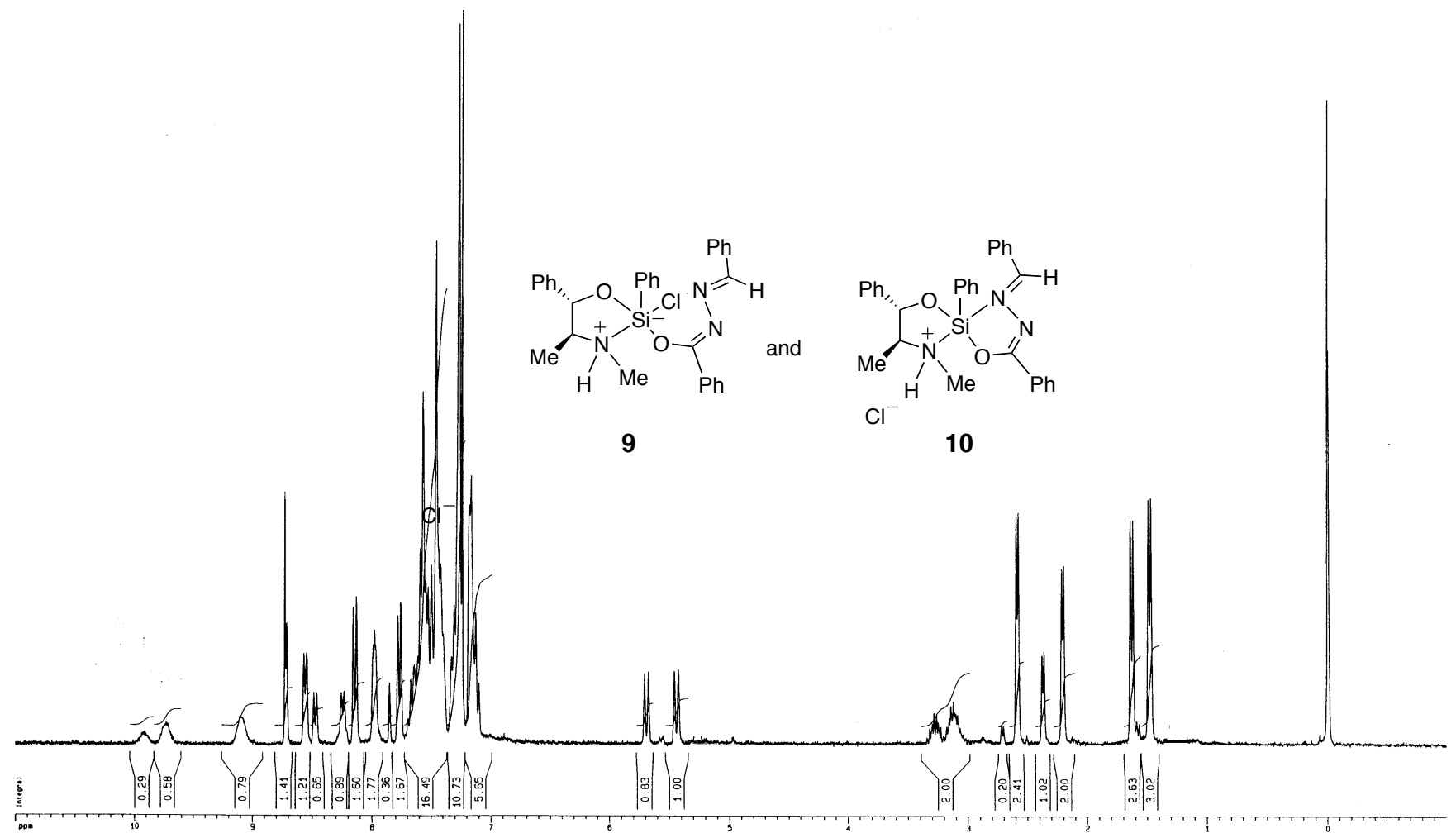


${ }^{1} \mathrm{H}$ NMR (400 MHz, $\mathrm{CDCl}_{3}$ ) spectrum of 11:

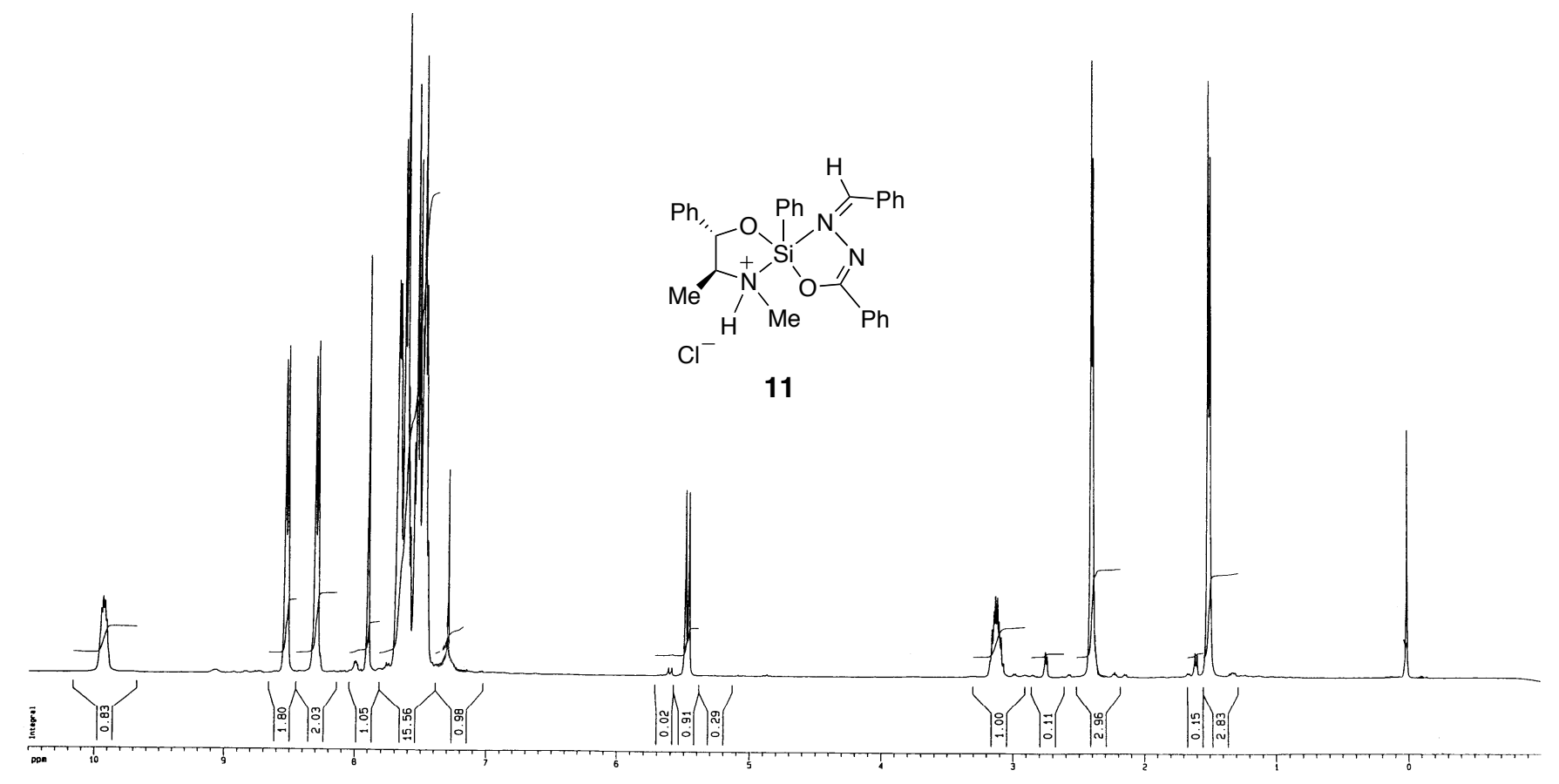

${ }^{29} \mathrm{Si} \mathrm{NMR}\left(60 \mathrm{MHz}, \mathrm{CDCl}_{3}\right)$ spectrum of silane 11:

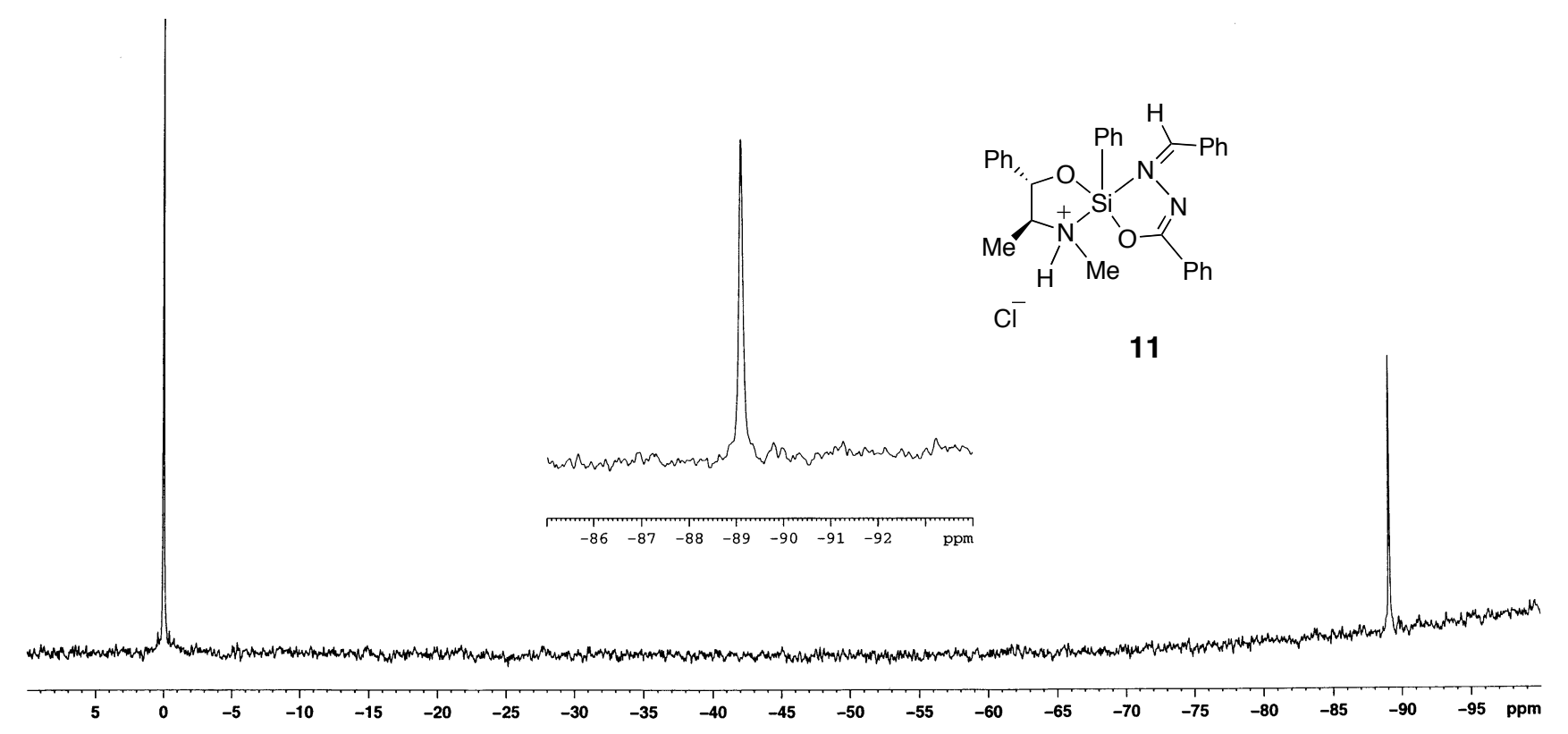

